\title{
A Survey of Multimedia Streaming in Wireless Sensor Networks
}

\author{
Satyajayant Misra, Martin Reisslein, and Guoliang Xue
}

\begin{abstract}
A wireless sensor network with multimedia capabilities typically consists of data sensor nodes, which sense, for instance, sound or motion, and video sensor nodes, which capture video of events of interest. In this survey, we focus on the video encoding at the video sensors and the real-time transport of the encoded video to a base station. Real-time video streams have stringent requirements for end-to-end delay and loss during network transport. In this survey, we categorize the requirements of multimedia traffic at each layer of the network protocol stack and further classify the mechanisms that have been proposed for multimedia streaming in wireless sensor networks at each layer of the stack. Specifically, we consider the mechanisms operating at the application, transport, network, and MAC layers. We also review existing cross-layer approaches and propose a few possible cross-layer solutions to optimize the performance of a given wireless sensor network for multimedia streaming applications.
\end{abstract}

Index Terms-Cross-layer mechanisms, medium access control, multimedia, video streaming, wireless sensor networks.

\section{INTRODUCTION}

A WIRELESS sensor network consists of sensor nodes that are powered by small irreplaceable batteries. These sensor nodes are densely deployed in the area to be monitored and sense and transmit data towards the base station [1]. A sensor network with both standard data sensors and video sensors, as illustrated in Fig. 1, is suitable for a variety of sensing applications, including surveillance [2]-[6].

The data sensors sense motion, sound, heat, or light to initially identify and locate the target. The video sensors may be triggered by the data sensors to provide video of the target, or may operate independently, to sense and transmit video of the environment.

Our focus in this survey is on the mechanisms for encoding the video at the video sensors and the real-time transport of the encoded video from the sensors to the base station (sink). Key challenges for the video coding in the sensors are the low power and computational capabilities of the sensor nodes. Key challenges for the transport are the real-time requirements of the bursty video traffic that needs to meet the periodic playout deadlines of the video frames as well as the several lossy

Manuscript received December 15, 2005; revised September 9, 2007. This research was supported in part by ARO grant W911NF-04-1-0385 as well as NSF grants Career ANI-0133252, CCF-0431167, and ANI-0312635. The information reported here does not reflect the position or the policy of the federal government.

Satyajayant Misra and Guoliang Xue are with the Department of Computer Science and Engineering, Arizona State University, Tempe, AZ 85287-8809 (e-mail: \{satyajayant, xue\}@asu.edu).

Martin Reisslein is with the Department of Electrical Engineering, Goldwater Center, MC 5706, Arizona State University, Tempe, AZ 85287-5706 (e-mail: reisslein@asu.edu).

Digital Object Identifier 10.1109/SURV.2008.080404. wireless hops between a sensor node and the base station. Unique characteristics of wireless sensor networks that can be exploited for meeting these challenges are $(i)$ that the base station has abundant power and computational capabilities for complex processing and $(i i)$ that the sensors capture typically redundant data of a given target (event) since the same event may be captured by multiple sensors.

We approach the challenges of multimedia streaming in wireless sensor networks on a per-layer basis, associating each challenge with the layer of the network stack at which it arises. We classify the solutions proposed at each layer based on the techniques used, giving their salient features and their merits and limitations. In addition, we indicate open issues at each layer. We also survey cross-layer approaches, including joint optimization, and identify their suitability for supporting multimedia applications in wireless sensor networks. We consider the visual quality of the video, measured, for instance, in terms of the peak signal-to-noise ratio (PSNR), as the primary performance metric. We note that other performance metrics may be of interest; e.g., in a network that processes and classifies the sensor data, classification accuracy is an important metric. We also note that we do not explicitly consider physical layer issues in this survey. However, we consider the impact of the unreliable wireless physical layer on the upper layers.

Related surveys on wireless sensor networks are the surveys by Chen and Varshney [7] and Younis et al. [8] on network and medium access control (MAC) layer mechanisms for supporting quality of service in wireless sensor networks. These existing surveys do not contrast the advantages and limitations of the different approaches, nor classify the approaches. Gurses and Akan [9] surveyed multimedia communication in wireless sensor networks with a primary focus on the application layer and the network layer, with limited consideration of the transport layer. However, they consider neither the MAC layer, nor cross-layer solutions. Our survey complements these existing surveys in that we $(i)$ comprehensively survey and classify the solutions proposed for multimedia communication at the application, transport, network, and medium access control (link) layers; (ii) survey cross-layer optimization and mechanisms for multimedia streaming; and (iii) outline directions for future research at each layer of the stack as well as directions for future cross-layer optimizations/streaming mechanisms.

This survey is structured as follows. In Section II, we present the requirements of a multimedia streaming application from the application layer perspective and classify the proposed application layer approaches. Following the same 


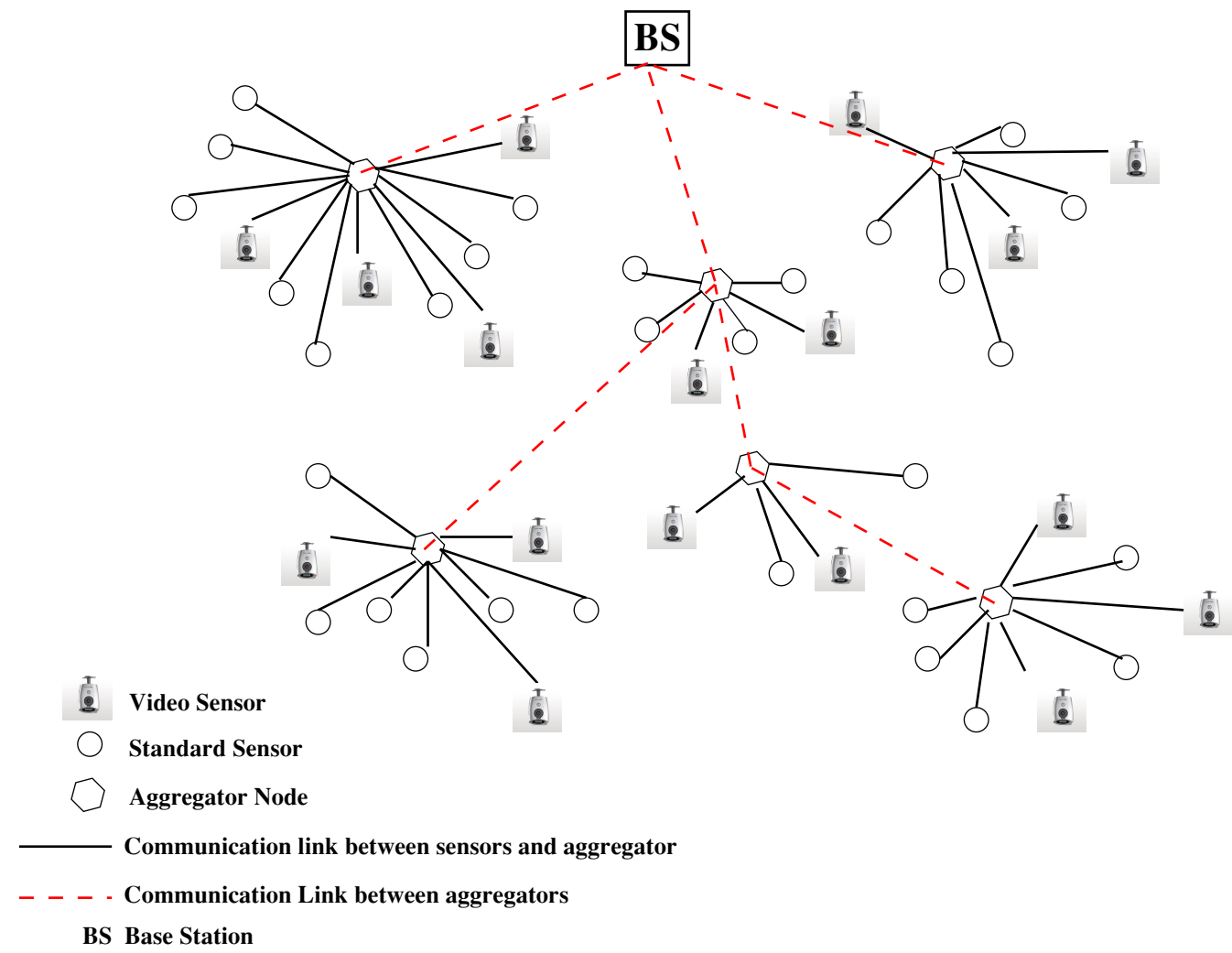

Fig. 1. Illustration of hierarchical wireless video sensor network structure: Video sensors capture and compress video of the target environment, either independently or triggered by standard data (e.g., motion, light, acoustic) sensors. The compressed video is streamed in real-time via aggregator nodes to the base station

pattern, we discuss in Sections III, IV, and V, respectively, the requirements of an multimedia streaming application from the transport layer, network layer, and MAC layer perspectives and survey the existing transport layer, network layer, and MAC layer solutions. In Section VI, we discuss cross-layer optimization approaches and streaming mechanisms for supporting multimedia streaming applications in a wireless sensor network. In Section VII, we conclude the survey and outline directions for future research.

\section{ApPlication Layer REQUiREMENTS AND MeChanisms For STREAMing APPLICATIONS}

A real-time streaming application is more demanding than data sensing applications in wireless sensor networks primarily due to its extensive requirements for video/audio encoding. The limitations of the sensor nodes require video coding/compression that has low complexity, produces a low output bandwidth, tolerates loss, and consumes as little power as possible. Most video sensors use embedded microprocessors, such as the Intel Strong ARM RISC [10], the XScale PXA-255 which is used in crossbow stargate motes [11], or the SAMSUNG S3C44B0X RISC processors [12]. As highlighted in Table I, these processors make the video sensors more powerful than basic data sensors, such as the TelosB nodes.

Despite being relatively powerful, the video sensors struggle to implement the compute-intensive motion estimation and compensation based predictive coding techniques used in the MPEGx or H.26x series [2]. For this reason, video sensors typically employ compression techniques that are founded on coding mechanisms for individual still images, such as JPEG and JPEG 2000 [13]. Some employed singlelayer compression techniques include added mechanisms to efficiently encode sequences of images (i.e., video) while avoiding motion estimation and compensation [2], [14]. We note, however, that there are recent efforts to scale down the computation complexity and power consumption of the motion estimation and compensation based MPEGx and H.26x compression techniques to make them suitable for wireless sensor networks [15], [16].

We do not discuss in detail audio coding and transmission. If a wireless sensor network can support image/video encoding and transmission it can typically support the less resource demanding audio coding and transmission. We briefly mention that the work of Zhang et al. [17] on acoustic streaming implemented on MicaZ motes [18] demonstrates acoustic streaming in wireless sensor networks.

We classify the application layer compression/encoding schemes in the literature on the basis of the coding paradigm and the compression scheme as illustrated in Figure 2. In principle, any combination of the two coding paradigms and three compression techniques is possible. However, to date, distributed source coding has primarily been investigated in the context of single-layer coding, while individual source coding has been examined in the context of all three compression techniques. We note that recently significant research has examined joint power optimization of source and channel coding to ensure that the source coding supports transmission with minimum channel error. This joint optimization across 
TABLE I

DIFFERENCE IN ABILITIES OF THE VIDEO AND STANDARD SENSORS

\begin{tabular}{|c|c|c|c|}
\hline & Stargate & Samsung S3C44B0X & TelosB \\
\hline Clock Frequency & $200 / 300 / 400 \mathrm{MHz}$ & $66 \mathrm{MHz}$ & $8 \mathrm{MHz}$ \\
\hline Architecture & 32 bit RISC & $16 / 32 \mathrm{bit}$ RISC & 16 bit RISC \\
\hline Memory & $\begin{array}{c}\text { 64 MB SDRAM } \\
32 \mathrm{MB} \text { Flash }\end{array}$ & $256 \mathrm{MB}$ & $\begin{array}{c}10 \mathrm{~KB} \\
1 \mathrm{MB} \text { Flash }\end{array}$ \\
\hline Cache & $\begin{array}{c}32 \mathrm{~KB} \text { data } \\
32 \mathrm{~KB} \text { instruction }\end{array}$ & $8 \mathrm{~KB}$ & $\begin{array}{c}\text { Data not } \\
\text { available }\end{array}$ \\
\hline Cost (\$) & 595 & 500 & 100 \\
\hline
\end{tabular}

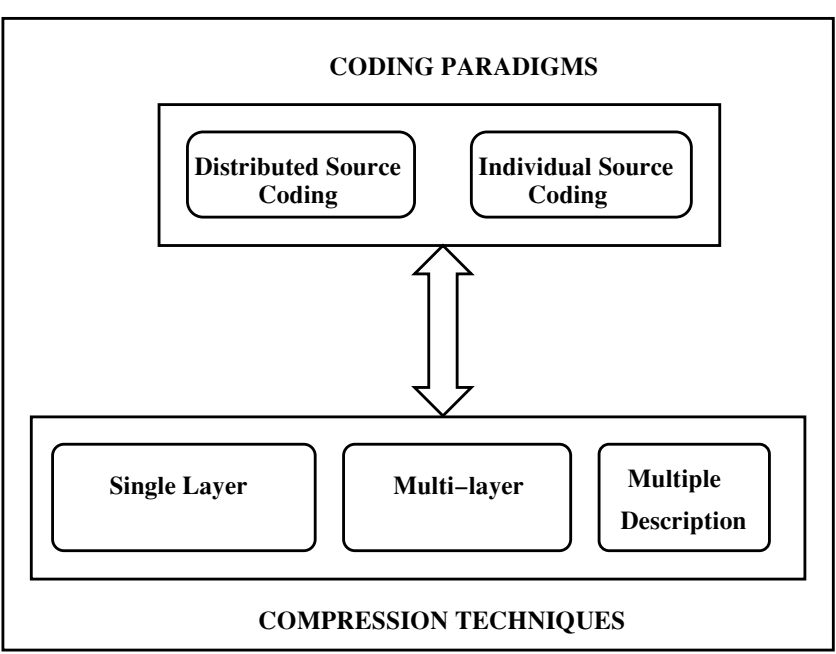

Fig. 2. Classification of application layer video coding mechanisms

multiple layers of the stack falls under the category of crosslayer optimizations and is discussed in Section VI.

\section{A. Coding Paradigms}

The coding paradigms that have been examined for multimedia wireless sensor network are distributed source coding and individual source coding.

1) Distributed Source Coding: Distributed source coding refers to the compression of multiple correlated sensor outputs from sensors with limited or no cooperation and joint decoding at a central decoder (at the base station or aggregator node). A more generalized setting for this problem is the multi-terminal source coding problem, also know as the CEO (Central Estimating Officer) problem [19]. Distributed source coding inverts the traditional one-to-many video coding paradigm used in most video encoders/decoders, such as MPEGx and H.26x. In the one-to-many paradigm, the encoders carry out complex encoding while the decoders are relatively less complex. In contrast, distributed source coding uses a manyto-one coding paradigm, while also swapping the complex encoder for a complex (heavy) decoder. Thus, the encoders at the video sensor nodes can be designed to be fairly simple requiring less resources, while the aggregator node or base station have the more complex decoder. Moreover, this allows the video sensor nodes to independently send data to the aggregator node/base station without exchanging information among themselves.

Initial theoretical performance bounds for distributed source coding were established by Slepian and Wolf [20], who proved

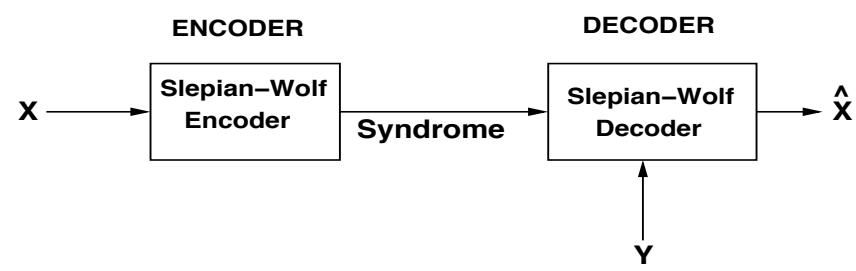

Fig. 3. Schematic Diagram of the Lossless Slepian-Wolf encoder-decoder

that for lossless compression, separate encoding of information with combined decoding at the decoder is as efficient as joint encoding. The Slepian and Wolf result could not be practically implemented until Wyner identified the relationship between distributed source coding and channel coding and suggested the use of linear channel codes for distributed source coding using Slepian-Wolf coding [21].

An example of distributed source coding using Slepian-Wolf coding is lossless source coding of correlated sources $(\mathcal{X}, \mathcal{Y})$ with side information at the decoder. In this lossless case, the decoder has access to the lossless side information $\mathcal{Y}$, and only obtains a compressed version of the information $\mathcal{X}$; this compressed version is referred to as the syndrome [22] of $\mathcal{X}$. Since $\mathcal{Y}$ and $\mathcal{X}$ are correlated, the decoder uses $\mathcal{Y}$ and the syndrome of $\mathcal{X}$ to output $\hat{\mathcal{X}}$ which is an estimation of $\mathcal{X}$. This results in a reduction of the total number of bits sent by the sources to the decoder.

For example, two sources A and B sending correlated information to a receiver $\mathrm{C}$ can use distributed source coding to reduce the total number of bits sent. One of the sources (say B) can send the complete information to $\mathrm{C}$ (the side information), while A sends only the syndrome (compression of its data). Receiver $\mathrm{C}$ can then use the data sent by $\mathrm{B}$ and the syndrome sent by A to estimate the data sent by A. With this technique, the bit rate sent by the sources is reduced as the number of sources sending correlated information to the decoder increases. Figure 3 shows the schematic diagram for a Slepian-Wolf encoder-decoder. The source coding may be done using various codes, such as trellis codes [23], turbo codes [24], and LDPC codes [25] to generate the syndrome bits.

Slepian-Wolf coding pertains to source coding in a lossless environment with side information $(\mathcal{Y})$. However, in sensor networks the transmission is lossy. Wyner and Ziv extended the work of Slepian and Wolf to establish information theoretic bounds for lossy compression with side information at the encoder [26]. In general, a Wyner-Ziv encoder can be thought to consist of two stages, a quantizer and a Slepian-Wolf encoder. Figure 4 shows a schematic diagram of the Wyner-Ziv 


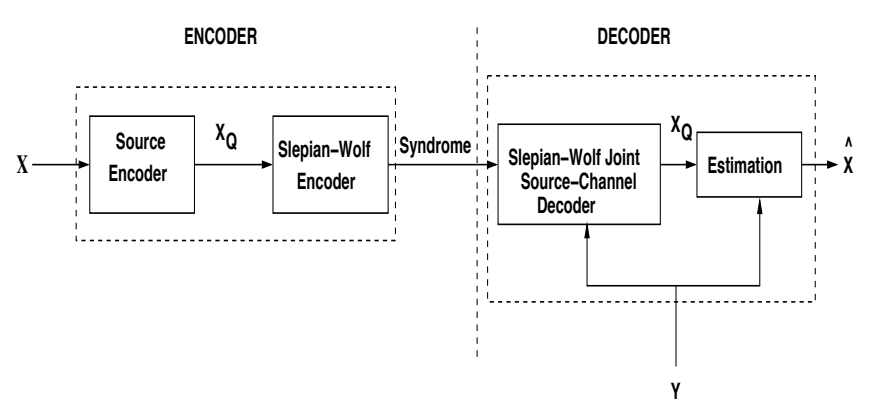

Fig. 4. Schematic Diagram of the Lossy Wyner-Ziv encoder-decoder

encoder/decoder. The quantizer at the source encoder separates the signals into mutually exclusive sets (bins) whereas, the Slepian-Wolf encoder performs channel coding on the quantized signal $X_{Q}$. At the decoder, joint source-channel coding is performed on the incoming signal using the side information $Y$ (complete sensed data sent by one of the sources), and then the signal is reconstructed with the side information such that the output has minimum distortion.

Wagner et al. [27] proposed a distributed source coding scheme for images captured by sensor nodes having overlapping fields of view. The approach uses a technique similar to stereo-image compression [28] to identify overlap in the images of neighboring sensor nodes. A low-resolution version of the overlapping region is sent by each sensor in the common neighborhood to the base station. The base station uses the super-resolution [29] technique to reconstruct a high resolution version of the overlapping region. Song et al. [30] presented an algorithm that uses transform coding of distributed sources and the geometric correspondence between sensor nodes of a given neighborhood to reduce the bit-rate required for image transmission. Majumdar and Ramachandran [31] proposed Power-efficient, Robust, hIghcompression, Syndrome-based Multimedia coding (PRISM), an error-resilient distributed source coding scheme based on coset codes. In PRISM, motion compensation is conducted at the decoder allowing for efficient compression exploiting the temporal redundancy between successive images (video frames) while keeping the coding effort at the sensor nodes low. Robustness to wireless channel losses is achieved through basing the coding of a given frame on the correlation statistics to a preceding frame, instead of the exact image data in the preceding frame. Simulation results indicate that PRISM achieves better video quality than the traditional H.263 codec for lossy wireless links. The use of distributed source coding to utilize the combined power of the sensor nodes in a neighborhood with overlapping fields of view appears to be an effective approach for energy-efficient image coding. However, there have not yet been testbed implementations of distributed source coding techniques.

The major advantage of using distributed source coding is energy savings, especially in dense wireless sensor networks. Energy savings occur due to the distributed processing by the sensor nodes sharing the overlapping fields of view as well as the reduction in the amount of the data transmitted from a neighborhood. The amount of data is reduced by sending the common overlapping region of the image only once, by one of the sensor nodes in the neighborhood, while the remaining sensor nodes send the coded syndrome. One of the drawbacks of distributed source coding is that it requires synchronization between the senders (encoders) because the decoder uses the time-correlated information to decode the packets. In addition, effective utilization of distributed compression requires knowledge of the correlation model of the data from the multiple sources, which is difficult to obtain. Also, distributed source coding based on Slepian-Wolf or Wyner-Ziv source coding with side information at the decoder requires some cooperation between the nodes. With present coding techniques, nodes cooperate in groups of two or three so that one can send the side information and the others send the coded information to reduce the rate to the Slepian-Wolf or Wyner-Ziv limit [32]. However, a better utilization of the correlation in the data is to utilize distributed source coding to code information from more than three sources. This problem has been hard to solve and no significant theoretical results have been obtained so far. Nevertheless, distributed source coding is a very promising mechanism for video/image coding in multimedia wireless sensor networks and we refer the reader to [33]-[35] for more details on the basics of distributed source coding.

2) Individual Source Coding: Single source coding is the traditional paradigm used in multimedia coding where each source codes its information independently of other sources. All existing testbed implementations of image/video coding and transmission fall under this category, see e.g., [2], [14], [36]-[38]. Individual source coding is popular due to its simplicity and because it does not require any kind of communication between the sources. However, it is apparent that in a wireless sensor network, individual source coding results in large redundancies. When there is a high concentration of sources in the vicinity of an event all these sources attempt to transmit similar data at the highest possible quality to the base station, resulting in a large number of copies of the same data. These copies result in no increase in the information content, but cause significant congestion and energy depletion. Fusing the correlated video data in the aggregator nodes has the potential to significantly reduce the amount of transmitted data. Effective video fusion mechanisms that are suitable for wireless sensor network are, however, largely an open area of research [39]-[41]. We believe that in the long run, the video coding in wireless sensor networks will rely on distributed source coding, individual source coding with fusion, or a combination of distributed source coding and fusion.

\section{B. Compression Techniques}

We categorize the compression techniques for multimedia transmission in a wireless sensor network as illustrated in Figure 2. We survey representative testbed implementations of single layered coding and multi-layered coding. We present the underlying concepts for multiple description coding for which there are no testbeds yet.

1) Single Layer Coding: JPEG with change or difference coding is a representative and widely studied single layer 
coding scheme for wireless sensor networks [2], [14], [36][38]. In this mechanism, a reference frame is periodically generated and transmitted by the sensor nodes towards the base station. In the time period between two reference frames, the sensor nodes transmit the differences/changes with respect to the reference frame. Chiasserini et al. [2] used JPEG with fixed point Discrete Cosine Transform (DCT), in place of the commonly used floating point DCT, to reduce computation complexity. In [37], the algorithm uses change detection on a reference to build a map of active regions. In this method, the current video frame is divided into blocks of $8 \times 8=64$ pixels. Only a subset of blocks in the frame are considered and only a subset of the pixels in each block are examined for changes in comparison to the corresponding pixels in the reference frame. Only the difference is encoded using JPEG and transmitted. The approach appears to provide MPEG-2 like quality for a fraction of the energy [37]. Pekhteryev et al. [38] demonstrated the feasibility of transmission of JPEG and JPEG 2000 images over a ZigBee network. Feng et al. [3], [14] proposed a video sensor platform composed of one of the first viable sensors. They used JPEG and differential JPEG and also had a priority based streaming mechanism for dynamic buffer management and video bit rate adaptation. The framework uses Stargate as well as the Intel Bitsy motes [10] and provides a frame rate of 34 frames/sec. The authors suggest that the implementation is energy efficient enough to be operated on 5 watt batteries, however, they do not specify the lifespan of each mote using these batteries.

The use of JPEG as the encoder along with difference coding has obvious advantages due to the low required processing power and the dramatic reduction in the total transmission energy in sending only the differences in comparison to the entire image. However, this scheme does not support aggregation (fusion), resilience and error control, nor joint source-channel coding, which attempts to combine error resilient source coding, channel coding, and error concealment [42]. This issue of resilient coding and error concealment can be addressed by using one of the many channel coding schemes, such as forward error correction (FEC) [43] or erasure correction (EC) codes [44]. Given the relatively larger size of multimedia data, the JPEG scheme may result in faster depletion of energy of the sensor nodes involved in data transmission to the base station when compared to schemes that incorporate aggregation. Difference coding makes it difficult to perform aggregation of the data as it requires information about the past context of the data to be stored at the aggregating nodes.

Another limitation of the JPEG based schemes with difference encoding is that the reference frames are critical for the video quality, as any errors in a reference frame, e.g., due to wireless channel errors, propagate to frames encoded with respect to the reference frame. This propagation problem also limits bit rate adaptations through adjustment of the quantization scales of the DCT coefficients as done in standard MPEGx/H.26x coding with rate control. Coding a reference frame with a lower bit rate at a lower (coarser) quality would reduce the quality of the frames that reference the coarse frame. On the other hand, rate adaptations of the encoded differences or wireless losses of difference/change information affects only individual frames, but provide a relatively small range of rate adaptation. The video bit rate can thus be adapted by assigning priority levels to the video data and dropping data according to priority levels [14], [45].

2) Multi-layer Coding: In this subsection, we review representative multi-layer coding schemes, including one scheme for which the energy consumption can be calculated, illustrating the trade-offs between energy consumption for compression and transmission. The multi-layer coding schemes examined for wireless sensor networks are typically based on JPEG 2000 [13] and accordingly employ wavelet transform based compression. DCT based compression can in general also produce layered encodings but has not been specifically studied for this purpose in the context of wireless sensor networks.

The multi-layer coding mechanism proposed in [46] uses wavelet based decomposition to create multiple bit-stream image encodings that are transmitted in small fragment bursts. The wavelet coefficients are grouped into multiple trees and encoded separately based on the set partitioning in hierarchical trees (SPIHT) algorithm [47]. Yu et al. [48] used JPEG 2000 to encode the image as a scalable bit-stream with multiple layers. These layers are of different quality and are optimal in the sense of rate distortion in joint source coding. The proposed scheme is named Joint Source Channel Coding and Power Control (JSCCPC) and uses Rate-Compatible Punctured Convolution (RCPC) codes for channel coding. Further, $\mathrm{Yu}$ et al. introduced the JSCCPC system composed of a source encoder, channel encoder, wireless transmitter, and the JSCCPC unit. The JSCCPC unit tunes the output from the coders based on the channel conditions.

Heinzelman et al. [49] proposed a mechanism for poweraware image transmission in wireless networks. They used a transceiver model [50] in which the power consumed to transmit a $k$-bit message over a distance $d$ is given by,

$$
E_{t x}(d)=k \cdot\left(\epsilon_{e}+\epsilon_{a m p} \cdot d^{\alpha}\right),
$$

where $\epsilon_{e}$ is the energy used by the circuit per bit, $\epsilon_{a m p}$ is the energy used by the amplifier per bit, and $\alpha$ is the path loss exponent. The power consumed in receiving $k$ bits is given by,

$$
E_{r x}=\epsilon_{e} \cdot k .
$$

In JPEG 2000, the compression ratio can be controlled by the transform level and the quantization level. Increasing the transform level can increase the compression ratio, however, it increases the energy consumption as well. The energy consumed per bit in compression as a function of the transform level $L$ was given in [49] as,

$$
\epsilon_{c}(L)=\gamma\left(f-4^{-L-1}\right), L=0, \ldots, L_{\max },
$$

where $\gamma$ is a device/implementation specific constant, $f$ is a constant determined by the quantization and entropy coding, and $L_{\max }$ is the maximum transform level. The values of the parameters for standard JPEG 2000 image coding with RF communications is given in Table II. From the above definitions, the energy required for compressing 1 bit of an image at a transform level of $L=2$ is $0.278 \mu \mathrm{J}$. While the energy required for transmitting and receiving 1 bit of the image over one $30 \mathrm{~m}$ hop is $0.19 \mu \mathrm{J}$. This required energy 
TABLE II

PARAMETERS FOR THE JPEG2000 BASED SCHEME IN [49]

\begin{tabular}{|c|c|}
\hline Parameter & Value \\
\hline$\alpha$ & 2 \\
\hline$\epsilon_{e}$ & $50 \mathrm{~nJ} / \mathrm{bit}$ \\
\hline$\epsilon_{a m p}$ & $0.1 \mathrm{~nJ} / \mathrm{bit} / \mathrm{m}^{2}$ \\
\hline$\gamma$ & $0.235 \mu \mathrm{J} / \mathrm{bit}$ \\
\hline$f$ & 1.195 \\
\hline
\end{tabular}

increases additively with the number of hops. For instance, if the hop length is 5 hops, the cost is $0.95 \mu \mathrm{J}$. The compression ratio was given by,

$$
\beta(L)=\frac{g_{1}}{g_{2}-g_{3}^{-L}}, L=0, \ldots, L_{\max },
$$

where $g_{1}=1.121, g_{2}=3.222$, and $g_{3}=3.542$ [49].

For a $320 \times 240$ pixel image with a depth of 8 bits per pixel we calculate the energy consumed in transmitting the uncompressed image over one hop as $0.117 \mathrm{~J}$. Compression reduces the image to 0.357 of its original size at an energy cost of $0.171 \mathrm{~J}$. The one-hop transmission of the compressed image requires $0.042 \mathrm{~J}$. Therefore, the total energy consumed, including compression, over 1-hop is $0.213 \mathrm{~J}$. Consequently, for 1-hop transmission the non-compressed image consumes less energy. If the transmission is over, say 5 hops, the transmission of the compressed image consumes a total of $0.381 \mathrm{~J}$ while the transmission of the uncompressed image consumes a total $0.585 \mathrm{~J}$, making the compressed image transmission preferable for multi-hop wireless sensor networks.

The advantage of multi-layer coding using JPEG 2000 based wavelet compression is the ability to use joint source-channel coding and adapt to the channel conditions. Depending on the channel conditions, the optimal number of layers can be selected and the optimal amount of channel coding can be done. A trade-off with this approach is the increasing energy consumption with increasing number of transform levels, i.e., increasing flexibility in rate adaptation. A limitation of multilayer coding with wavelet compression is that this technique has so far primarily been studied in the context of coding individual still images in wireless sensor networks. While there exist general multi-layer wavelet compression schemes with motion compensation, the investigation of multi-layer coding with wavelet compression in conjunction with effective and energy-efficient motion compensation to exploit the temporal redundancy between successive images is largely an open area in wireless sensor networks. Further, with the existing multilayer approaches it is difficult to aggregate video data in the vicinity of the event and along the path to the base station. This is because the variable coding and redundancy levels necessitate an adaptive aggregation mechanism, thus requiring both more memory and computing power.

3) Multiple Description Coding: One of the disadvantages of the layered encoding schemes is that losing packets carrying a lower layer renders the received higher layer packets useless. Consequently, the packets carrying lower layers should be transported with higher priorities over the network. The technique of multiple description coding (MDC) [51] overcomes this problem. An ideal multiple description coder creates two bit streams of equal importance and the two streams are sent across two separate channels. To illustrate, let the bit rate of channel 1 be $R_{1}$ and that of channel 2 be $R_{2}$, then the total bit rate of the MDC based coder is $R_{1}+R_{2}$. At the receiving decoder, either of the following three cases can occur. Both the descriptions reach the decoder, only channel 1 reaches the decoder, or only channel 2 reaches the decoder. In essence, an MD decoder may be conceptualized to consist of three decoders, each corresponding to one of the three outlined cases. The encoder considers the possibility of either of the three situations occurring. The decoder that receives both channels is the central decoder, while the other two decoders that receive one channel are known as the side decoders. The central decoder produces a high quality re-construction of the video/image, while the other two decoders receiving only one channel each produce acceptable descriptions, however, with lower qualities.

The efficiency of a Multiple Description (MD) encoder is obtained using the Redundancy Rate Distortion (RRD) curve [52]. If the rate-distortion of the best single description coder is defined as $D_{0}$, and $R$ is the corresponding minimum required rate, then the redundancy of a MD coder is given by, $\rho=R_{M D}-R$, where $R_{M D}$ is the rate required by the multiple description encoders such that the central MD coder has a rate distortion $D_{0}$, and the side decoders have a rate distortion $D_{1}$. If $\rho$ is defined as the extra bit rate required for reducing the distortion of the side decoders $D_{1}$, then $\rho\left(D_{1}, D_{0}\right)$ is defined as the RRD function. The RRD function is the additional rate that is required to achieve a desired rate distortion $D_{1}$ at the side decoders and $D_{0}$ at the central decoder.

Path diversity of the packets along with MDC improves the robustness of video transmission [53]. A combination of path diversity and MDC provides robust end-to-end communication and high bandwidth in radio networks [51], [54].

MDC has some inherent shortcomings. At lower transmission rates, MDC is not as efficient as layered coding, which provides better quality for a given low encoding rate. Hence, the performance of MDC may be lower than that of layered coding for low transmission rate wireless sensor networks. However, MDC gives typically better performance than single layered coding, which does not provide error resilience, i.e., when the single layer transmission is missing the video is interrupted. In theory, the use of more descriptions improves performance. However, in wireless networking practice the performance improvement is limited due to the overhead for the multiple streams, such as headers for the packets of each stream [55]. Therefore, an optimized MDC needs to consider the header size of each description as well as the ratio of the header to the payload.

\section{Summary}

Due to the lack of comprehensive comparison studies and testbed implementations of the different coding paradigms and compression techniques, we can not provide quantitative comparisons. Clearly, such comprehensive quantitative comparisons would shed significant insight into the relative tradeoffs between the different coding approaches and should be a high priority for future research. We are limited to providing in Table III the main qualitative pros and cons of the compression 
TABLE III

COMPARISON OF SCHEMES AT THE APPLICATION LAYER

\begin{tabular}{|c|c|c|}
\hline Scheme & Advantages & Drawbacks \\
\hline $\begin{array}{c}\text { Single layer coding [2], [14] } \\
{[3],[36]-[38]}\end{array}$ & $\begin{array}{c}\text { Low processing power, } \\
\text { reduction in total energy }\end{array}$ & $\begin{array}{c}\text { Aggregation, resilience not supported, } \\
\text { rate adaptation limited }\end{array}$ \\
\hline Multi-layer Coding [5], [48], [49] & Adapts to channel conditions & $\begin{array}{c}\text { Cannot exploit the } \\
\text { redundancy in the network }\end{array}$ \\
\hline MDC [51]-[54] & $\begin{array}{c}\text { Robust end-to-end } \\
\text { communication }\end{array}$ & $\begin{array}{c}\text { Performance not good at } \\
\text { lower transmission rates }\end{array}$ \\
\hline
\end{tabular}

schemes. The single-layer encoding schemes have limited rate control ranges, and hence require the use of error correction or retransmission at the transport layer to ensure reliable transport over channels with widely varying bit rates. An attractive alternative is scalable video compression with feedback, whereby the sources adjust the number of transmitted layers in response to changing network conditions. One such mechanism proposed for the wired Internet is Scalable Video for Internet Protocol (SVIP) [56], which also produces packets of equal visual importance. A similar protocol would be of great utility for a wireless sensor network that supports multimedia traffic, this could be a viable direction for future research on scalable coding.

\section{Security and Privacy Issues for Streaming Applications}

Similar to other wireless sensor networks, security issues, such as confidentiality, authentication, and integrity are pertinent in a wireless sensor network supporting multimedia streaming applications. In this section we first give an overview of the main commonalities and differences between security and privacy mechanisms in data and multimedia wireless sensor networks, and then briefly review encryption schemes for multimedia wireless sensor networks. Many schemes have been proposed in the literature, to perform key assignments and set-up, ranging from deterministic to randomized schemes [57]-[62]. These schemes can be applied in the wireless sensor networks we study. In addition, the mechanism of message authentication codes used in all communication networks for integrity and authentication is readily usable [63]-[65].

The area where multimedia streaming applications are different from other applications in wireless sensor networks is in the usability of encryption techniques to ensure confidentiality. In a wireless sensor network, the public key cryptography schemes are not suitable because of their high power and computation requirements. The standard symmetric encryption schemes, such as DES and AES, are commonly used. However, these schemes are unsuitable for multimedia data. Multimedia data is generally larger in size and use of these symmetric encryption schemes has memory and computation requirements that are unsupportable by the sensor nodes.

Depending on the nature of the information gathered by video sensors there may arise issues of privacy. For instance, if the video sensors are used to monitor the vehicles in a parking lot, they may acquire other corresponding images, such as the images of the license plates of the vehicles, or that of the occupants of the vehicles. This information is sensitive and might intrude on the privacy of the users of the parking lot. If the base station cannot be trusted with hiding/removing the sensitive information then the video sensors have to do that. Thus, more sophisticated image processing algorithms are needed. This is an open area of research.

Denial of service and service disruption by malicious adversaries can become a severe problem in the wireless sensor network supporting multimedia streaming applications. An adversary can corrupt or inject false multimedia packets and these packets may be forwarded all the way to the base station where they are found to be unusable. However, the intermediate forwarding sensor nodes would be drained much faster of battery energy.

To round out this overview of security and privacy issues for streaming applications, we briefly review the encryption schemes that have been proposed for multimedia data. As mentioned, standard symmetric encryption schemes are unsuitable for encryption of multimedia data since the multimedia data is generally larger in size than standard sensor nodes data and also because its real time nature requires faster encryption. However, multimedia encryption can be lossy in comparison to text data encryption which is generally lossless. All existing multimedia encryption schemes are based on three mechanisms:

- Position Permutation: The data bits are permuted according to some predefined scheme [66], [67]. This is a simple and fast mechanism. However, it does not have strong security guarantees.

- Value transformation: The data value is transformed using some type of transformation [68], [69]. At the receiver the transformation is reversed to obtain the real data. This mechanism has low computational complexity and low hardware implementation cost.

- Combination: This technique uses a combination of both position permutation and value transformation and has a very high data security [70], [71].

Most of the proposed schemes do not have any restrictions on the processing or memory power of the encrypting/decrypting systems. Although, the mechanisms proposed have low complexity and high speed their suitability for wireless sensor networks is an open question. In addition, the development of modified DES or AES schemes that have improved efficiency and speed is also an area that needs to be explored.

\section{TRANSPORT LAYER REQUiREMENTS AND MeChanisms FOR STREAMING APPLiCATIONS}

\section{A. Transport Layer Functionality in Wireless Sensor Networks}

Traditionally, the transport layer is responsible for assuring end-to-end reliability of the data transfer and congestion control in a network. In wireless sensor networks, the notion 
of end-to-end packet delivery reliability is in most cases unnecessary [72]. Most wireless sensor networks have a dense deployment of sensor nodes, and therefore a significant amount of correlation in the data from an event locality, as all sensor nodes in the locality observe the same event. Hence, instead of end-to-end packet based reliability, the notion of end-to-end event reliability is a more practical paradigm. Event reliability entails the successful transmission of a prescribed number of packets from the event area to the sink, within a prescribed interval of time, rather than successful transmission of all the packets from all the sensor nodes. In addition, to supporting multimedia traffic, the transport layer is required to meet challenges, such as bounded delay and jitter in data delivery, minimum bandwidth availability, multiple data priorities, and session maintenance.

The transport protocols used in the Internet, such as TCP, SCTP [73], and RTP/RTCP [74] cannot be used in a wireless sensor network. Transport protocols for wired networks or the Internet are complex and the underlying networks assumptions, such as no interference and packet loss resulting predominantly from congestion rather than the noisy medium, do not apply in the wireless setting. Also, the congestion in a wireless sensor network is burstier as the sensor nodes may not have any data to transmit for most of the time, however, when an event occurs all sensor nodes in the neighborhood start transmitting. As a result of these differences, the use of Internet/wired network transport protocols results in throughput degradation and significant energy inefficiencies [75]. Furthermore, the transport protocols developed for general ad hoc wireless networks (both extensions from wired transport protocols and novel approaches), such as [76]-[78] cannot be applied to a wireless sensor network mainly because $(i)$ the underlying assumption of reliability is different (end-to-end and not event-to-sink); ( $i$ i) most of the communications in a wireless sensor network are directed to a few nodes in the network (sinks) in contrast to general wireless networks where any node can become the receiver of a communication; and (iii) the communication paradigm in ad hoc networks does not support aggregation.

We proceed to review the transport protocols that have been developed for wireless sensor networks in recent years. However, we note that presently there does not exist any transport protocol in the literature specifically for supporting multimedia traffic in wireless sensor networks. Therefore, we review general transport protocols for wireless sensor networks and examine their suitability for supporting multimedia traffic.

A few protocols have been developed for providing reliable sink to sensor nodes data transport, such as Trickle [88], PSFQ [89], and GARUDA [90]. In this survey, we do not consider the sink to sensor nodes communications, but instead concentrate on the many-to-one sensor nodes to sink communication that makes up the bulk of all packet transmissions. The many-to-one transport protocols proposed for wireless sensor networks can be classified into three classes as illustrated in Figure 5.

\section{B. Protocols Focused on Congestion Control}

The protocols performing congestion control attempt to reduce congestion by detection, notification, and subsequent rate adjustments. The congestion is detected at sensor nodes along the path from the sender to the base station or by the base station. When congestion is detected, the detecting node(s) transmit congestion notification messages in the general direction of the sources. The notification may be explicit, that is, sent as a separate control packet, or implicit, that is, embedded within future data packet(s). Upon receiving the congestion notification, the source adjusts its transmission rate to prevent further congestion. Also, many solutions use packet caching at intermediate nodes in the network to reduce the energy expenditure in end-to-end transfer and to reduce latency resulting from packet loss due to congestion.

Hull et al. examined Fusion [79], a combination of three techniques, each at different layer of the protocol stack. They control hop-by-hop traffic flow, limit source traffic dependent on the presence of transit traffic in the network, and prioritize medium access control (MAC). The congestion control algorithm is based on the concept of back-pressure, with a sensor node $u$ that observes a high occupancy of its transmission queue signaling to the neighbors in its omnidirectional radio transmission range by setting a congestion notification flag in the packets that it forwards towards the base station. This serves as an implicit notification of congestion to the sensor nodes that are forwarding their traffic to the base station through $u$. These forwarding sensor nodes reduce their data rate when they see the flag set and set the congestion flag in the future packets that they transmit. This process ensures that the source of the packets eventually becomes aware of the congestion and reduces its transmission rate.

The rate control is performed by a mechanism similar to the token bucket mechanism. The scheme assumes that the data rate of the sensor nodes is the same. Each sensor node monitors the amount of traffic its parent (the sensor node that forwards its packets towards the base station) in the routing tree transmits, to identify the number of sources $N$ whose packets the parent forwards. A sensor node accumulates one token in its bucket every time that the parent transmits $N$ packets. The considered sensor node can only transmit if it has at least one token in its bucket. The MAC scheme used is CSMA. To implement a prioritized MAC, the authors propose to make the backoff window size of each sensor node a function of its congestion state. Thus, a sensor node that is more congested, that is, has more packets to send, is likely to get the chance to transmit more often. Simulation results indicate that a fusion of the three outlined techniques can improve transmission efficiency by three times under realistic workloads.

There are some possible shortcomings in this scheme when it comes to supporting multimedia traffic. In a large wireless sensor network the back-pressure resulting from implicit notification takes a long time to reach the source(s) of the traffic. Given the bursty nature of multimedia traffic, in the time it takes for the sources to get the notification, the traffic from the source(s) can overwhelm the forwarding sensor nodes in the network. The rate control scheme assumes that all sensor 


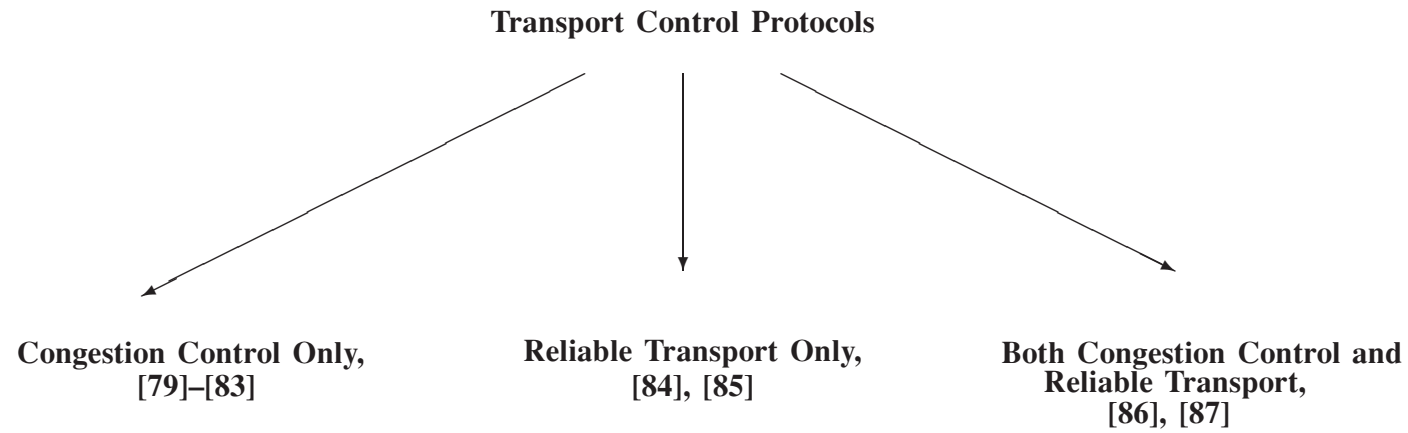

Fig. 5. Classification of Transport Layer Techniques

nodes have the same amount of traffic to send. This is not the case when distributed source coding is used, where a few of the sensor nodes transmit the side information (more data) whereas others transmit the syndromes only. The priority scheme is based on congestion, however, in multimedia traffic there can be packets that have different priorities, such as packets carrying the base layer of a multi-layer coding. Hence, the MAC protocol should support packets of varying priorities and dispatch them so as to meet their delay bounds.

Wan et al. [80] proposed COngestion Detection and Avoidance (CODA) which is comprised of three mechanisms: $(i)$ receiver-based congestion detection; (ii) hop-by-hop backpressure without feedback; and (iii) a feedback based multisource regulation. The mechanisms of FUSION [79] are very similar to CODA, hence CODA suffers from the same shortcomings. Ee and Bajcsy [81] proposed CCF, a transport control protocol that controls congestion and also improves fairness in the network. The scheme relies on the routing tree structure to equally assign the available bandwidth at the root of a routing sub-tree among its adjacent downstream links, thus creating an equal distribution of bandwidth. CCF also prevents congestion, as the senders do not exceed the assigned bandwidth. This scheme uses a simplistic definition of fairness, which is not applicable to multi-priority traffic. The scheme requires significant enhancements to support multimedia traffic having multiple priorities and different bandwidth requirements.

Wang et al. [82] proposed a node Priority-based Congestion Control Protocol (PCCP). The main idea of the protocol is prioritizing each sensor node to reflect its importance. Each sensor node is assigned a priority based on the amount of traffic originating at it and also the amount of traffic it is forwarding. PCCP performs congestion detection using a parameter referred to as the congestion degree, which is defined as the ratio between the mean packet service time and the mean packet inter-arrival time at a sensor node. If the ratio is larger than one, the sensor node experiences congestion. Congestion notification is implicit, with a sensor node attaching its congestion degree in the packets it forwards. Its neighbors can hence identify if the sensor node is experiencing congestion. The congestion algorithm also performs priority based rate control at each sensor node, based on its priority and congestion degree. Both link level and node level congestion is controlled. This scheme suffers from the same drawbacks as the ones mentioned above.
In the Siphon study [83], Wan et al. indicated that the use of techniques, such as packet dropping or rate control for controlling congestion affect the fidelity of the application at the sink. To improve application fidelity while controlling congestion, the authors suggest the use of virtual sinks to siphon off the data from areas in the network with a high event traffic load. These virtual sinks are deployed randomly or selectively in the network. Wan et al. propose algorithms for virtual sink discovery and selection, congestion detection, and traffic redirection to relieve congestion in the network. The congestion control algorithm at the virtual sinks runs on top of an underlying congestion control algorithm, such as CODA or Fusion. The idea is promising, however, it still requires the use of an effective underlying congestion control algorithm that can support multimedia traffic.

All the proposed congestion control protocols have similar shortcomings. They do not support multiple priorities at the application level. They use implicit notification, which requires fewer packet transmissions, but takes longer to effect congestion control. Also, the notion of fairness that all the protocols support is very simple, the extension to support the notion of fairness in the event of traffic with multiple data priorities originating from a single or multiple sources is nontrivial and is unaddressed.

\section{Protocols Focused on Reliable Transport}

The protocols designed for reliable transport ensure errorfree and complete transmission of data packets from the sources to the base station. Most reliable transport protocols in wireless sensor network use hop-by-hop packet recovery mechanism instead of an end-to-end mechanism to conserve energy. Zhang et al. [84] identified the disadvantages with general hop-by-hop recovery mechanisms and designed a Reliable Bursty Convergecast (RBC) scheme as an improvement. The scheme includes a window-less block acknowledgment scheme for reliable data transport. It was shown to increase channel utilization, reduce the probability of loss in acknowledgment, and hence, remedy the problems with hop-by-hop recovery mechanisms. The scheme appears to be effective for bursty traffic consisting of simple sensor data. However, the suitability of RBC for multimedia traffic, which requires more bandwidth, may have more intense traffic bursts, and is jitter prone, has not been studied.

Reliable Multi-Segment Transport (RMST) was proposed by Stann and Heidemann [85] for providing a reliable transport 
protocol for directed diffusion [91]. It provides segmentation and reassembly of data packets and also guarantees delivery of all packets from each source to the sink. Reliability for all packets in the network is inherently wasteful in the many-toone data transmission environment as it does not exploit the redundant nature of the traffic. Thus, in general, RMST is not a suitable mechanism for wireless sensor networks.

\section{Protocols Providing Congestion Control and Reliability}

The STCP [86] and ESRT [87] protocols attempt to provide transport solutions that simultaneously perform congestion control and reliable data transport. The STCP protocol proposed by Iyer et al. [86], provides variable degrees of reliability, congestion detection and avoidance, as well as support for multiple applications. It is based on a concept very similar to sessions [92]. A sensor node that wants to transmit data to the base station sends a session initiation packet. The base station on receiving the packet gets information about the number of flows originating from the source, whether the data is continuous or event-driven, the rate of transmission, and the required reliability. The base station then acknowledges the receipt of the session initiation packet to the source, which starts transmitting as soon as it receives the ACK. The base station ACKs every packet received or NACKs every packet whose timer expires without receipt. STCP controls congestion with a modified random early detection (RED) [93] mechanism. As soon as congestion is detected by an intermediate sensor node it sets the congestion notification bit in the packet that it forwards towards the base station. The base station on receiving a packet with the congestion notification bit set sends an explicit notification to the source.

The STCP protocol does not appear to be scalable in wireless sensor networks with a large number of hops from the source to the base station. In networks with a large number of hops, there would be a large latency between the onset of congestion in a neighborhood and the notification of the source. This large latency combined with the high data rates of multimedia streaming applications could cause very severe congestion before the congestion avoidance can even commence. Also the mechanism of ACKing each received packet and NACKing each timed-out packet is bandwidth and energy intensive.

The concept of event centered end-to-end reliability was first proposed by Sankarasubramanian et al. [87] as event-tosink reliability. The reliability centers on the notion that the sink is interested in the features of the event, obtained from the collective data from the sensor nodes in the region and hence, obtaining a prescribed number of packets from the sensor nodes is more important than reliably obtaining every packet. The authors proposed the use of a reliability indicator $r_{i}$ to measure the event reliability based on the number of packets received by the sink in given time period $t_{i}$. The observed event reliability $r_{i}$ in interval $t_{i}$ is the number of packets received by the sink in $t_{i}$. The desired event reliability $R$, is the number of packets necessary for reliable event detection. $R$ is an application dependent parameter. $r_{i}$ is calculated by the sink from the information received from the packets during $t_{i}$. If $r_{i} \geq R$, then the event is reported reliably, else it is not. The
ESRT protocol also uses the reporting rate $f$, defined as the number of packets sent out by a sensor in the event area per unit time. It attempts to configure the value of $f$ such that the desired rate $R$ is achieved with minimum energy utilization while controlling congestion.

The ESRT protocol is designed for a standard wireless sensor network in which the sensor nodes report data readings. The usefulness of the protocol for transport of multimedia traffic needs to be studied from several perspectives. For instance, depending on the used data encoding scheme some of the packets may have higher priority than others and contribute more towards event detection. The ESRT protocol needs to be configured to accommodate unequal packet contributions to event detection. Also, multimedia data is generally fragmented into many packets. Hence, a single event report from a source may be split into several transmitted packets. This requires a modified definition of the observed reliability to consider the number of complete reports received by the sink instead of packets. In addition, the increased congestion due to the nature and size of multimedia traffic and jitter of the traffic needs to be addressed.

\section{E. Summary}

From our evaluation of the transport layer protocols we find that the existing protocols are not well suited for supporting multimedia traffic in wireless sensor networks. A summary of the drawbacks of the protocols is given in Table IV. Among the examined protocols, the STCP and ESRT protocols come closest to being able to support multimedia traffic However, many requirements, such as support for multipath routing protocols (requires significant state maintenance), as well as data segmentation and accurate reassembly have not been addressed, while the mechanisms for congestion control need to be improved.

In summary, the following aspects need to be addressed by future transport solutions: capability of handling multiple priorities; ability to perform fast congestion control through fast notification of the sources; ability to handle high data rates along with jitter; mechanisms for creation and maintenance of event-driven sessions; accurate data segmentation and reassembly; and support for multipath routing protocols.

We believe that the use of two transport control protocols at the transport layer, one for congestion control and the other for reliable transport, is not the correct approach as it may be sub-optimal. A single protocol that jointly handles congestion control and reliable transport and is jointly optimized is more suitable.

\section{Network LAyer Requirements And MeChanisms FOR STREAMING APPLiCATIONS}

The network layer is important for QoS support for realtime multimedia streaming application in wireless sensor network because of two main reasons: $(i)$ it is responsible for providing energy efficient and stable routes that meet the endto-end QoS guarantees, and $(i i)$ it serves as an intermediate for the exchange of performance parameters between the MAC and the application layer. Multi-hop transmission is a commonly preferred mechanism for routing in a wireless 
TABLE IV

COMPARISON OF SCHEMES AT THE TRANSPORT LAYER

\begin{tabular}{|c|c|c|}
\hline Scheme & Approach Used & Drawbacks \\
\hline \multicolumn{3}{|c|}{ Congestion Control Mechanisms } \\
\hline FUSION [79] & $\begin{array}{l}\text { Combination of hop-by-hop } \\
\text { flow control, rate-limited traffic, } \\
\text { and prioritized MAC }\end{array}$ & $\begin{array}{c}\text { Does not support multiple packet priorities, } \\
\text { implicit notification slow, assumes all sensor nodes } \\
\text { have same amount of data }\end{array}$ \\
\hline CODA [80] & $\begin{array}{l}\text { Receiver based congestion detection } \\
\text { and multi-source regulation with } \\
\text { feedback }\end{array}$ & $\begin{array}{c}\text { Does not support multiple packet priorities, } \\
\text { implicit notification provides slow } \\
\text { congestion control. }\end{array}$ \\
\hline CCF [81] & $\begin{array}{l}\text { Congestion control based on routing tree } \\
\text { with a notion of fairness }\end{array}$ & $\begin{array}{c}\text { Simplistic notion of fairness, cannot handle } \\
\text { multiple data priorities }\end{array}$ \\
\hline PCCP [82] & $\begin{array}{l}\text { Congestion control based on sensor } \\
\text { node priority and congestion degree }\end{array}$ & $\begin{array}{l}\text { Congestion notification implicit, } \\
\text { does not support packet priorities }\end{array}$ \\
\hline Siphon [83] & $\begin{array}{l}\text { Virtual sinks to improve fidelity affected by } \\
\text { packet dropping and rate control }\end{array}$ & $\begin{array}{l}\text { Dependent on an effective congestion control } \\
\text { algorithm as the base. }\end{array}$ \\
\hline \multicolumn{3}{|c|}{ Reliable Transport Mechanisms } \\
\hline RBC [84] & $\begin{array}{l}\text { Window-less block ACK scheme } \\
\text { to handle moderately bursty traffic }\end{array}$ & $\begin{array}{l}\text { Support for burstier and jitter prone } \\
\text { multimedia traffic not studied }\end{array}$ \\
\hline RMST [85] & $\begin{array}{l}\text { Correct segmentation, reassembly } \\
\text { and delivery guarantee for all packets }\end{array}$ & Provides reliability for all packets, hence is wasteful \\
\hline \multicolumn{3}{|c|}{ Both Congestion Control and Reliable Transport Mechanisms } \\
\hline STCP [86] & $\begin{array}{l}\text { Based on sessions, congestion } \\
\text { control using RED mechanism }\end{array}$ & Not scalable to large networks \\
\hline ESRT [87] & $\begin{array}{l}\text { Event-to-sink reliability and } \\
\text { congestion control through the } \\
\text { use of the reporting rate }\end{array}$ & $\begin{array}{c}\text { Does not consider multiple priorities, or reliability } \\
\text { in fragmented packets. Handling of large packets or } \\
\text { jitter not studied. }\end{array}$ \\
\hline
\end{tabular}

sensor network because short-range wireless transmission that are relayed by several intermediate nodes tend to save energy and increase overall network lifetime [1]. However, multihop routing tends to increase the delay due to queuing and processing at each intermediate node. Generally, there is an energy-latency trade-off in that the larger the number of hops, the smaller the required energy, but also the larger the expected end-to-end delay.

QoS aware routing has been a widely studied topic in the area of wireless ad hoc networks. However, most of the proposed solutions are only useful in a mobile ad hoc networks setting [94]-[97]. Given the distinctions between wireless sensor networks and mobile ad hoc networks, as detailed in Section III-A, the QoS aware protocols for wireless mobile ad hoc networks can typically not be directly applied to wireless sensor networks. Therefore, research has begun to develop specific solutions for providing QoS at the network layer in wireless sensor networks, which we survey in this section.

We categorize the existing approaches for providing network layer QoS in wireless sensor networks into approaches focused on timeliness assurances and approaches focused on reliability, as illustrated in Figure 6, and contrast the main features of the approaches in Table V.

\section{A. Timeliness}

Based on the supported packet priorities, we classify the timeliness approaches into three subcategories as follows. In the No priority category, the real-time packets have a higher priority than the best effort traffic, however all the real-time packets have the same priority. In the Static priority category, all the packets for a given real-time flow have the same priority. The Differentiated priority category refers to the case where all packets can have different priorities.

Akkaya and Younis [98] proposed an energy aware QoS routing protocol (EAQos) falling in the no priority category. The EAQos protocols is designed for the real-time traffic generated by a wireless sensor network consisting of image sensors. The cost of a link is defined to capture communication parameters, such as the residual energy in the nodes, the transmission energy, and the error rate. All traffic is divided into the best effort and real-time classes. A class based queuing approach is used that allows service sharing between the two classes of traffic. The protocol finds multiple paths from a source to a destination, using an extended version of Dijkstra's algorithm. The source chooses a route that meets the endto-end delay requirements and then forwards the packet to the next-hop neighbor on the route. The scheduling algorithm ensures that the best effort traffic does not starve. The merit of this algorithm lies in the fact that it provides guarantees for best-effort transmission while at the same time trying to maximize the real-time traffic throughput. The main drawback of this approach is that it does not support multiple priorities for the real-time traffic. For a multimedia streaming application, different packets may have different QoS requirements, which can not be supported with the EAQos routing protocol. Also, the algorithm for calculating multiple paths requires complete knowledge of the network topology at each node, which limits the scalability of this approach.

He et al. [99] proposed a static priority routing protocol named SPEED to provide soft real-time guarantees for communication in a sensor network. SPEED uses stateless nondeterministic geographic forwarding as the primary routing mechanism. Geographic forwarding routes packets without requiring end-to-end path set-up. In SPEED, the network is supposed to support a given maximum delivery speed for every admitted packet. The term delivery speed is defined by $\mathrm{He}$ et al. as the rate (in $\mathrm{m} / \mathrm{s}$ ) at which the packet travels along the straight line from the source towards the destination. This definition of speed makes the end-to-end delay in the network proportional to the distance (in $\mathrm{m}$ ) between the source and the destination. Any session that wants a speed higher than 


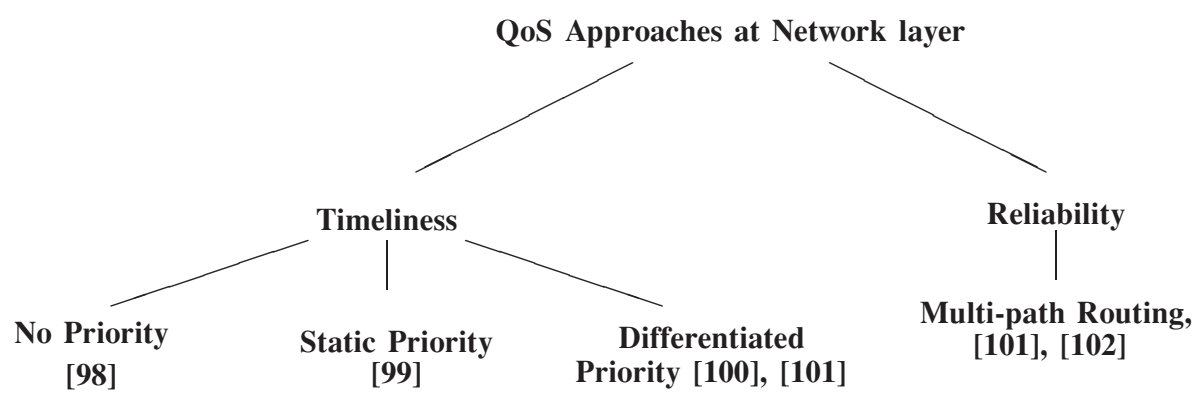

Fig. 6. Classification of Network Layer QoS approaches

the maximum delivery speed is not admitted. The routing algorithm calculates the transmission delay for a packet using the end-to-end distance and the maximum delivery speed. If the session requires a lower delay, then it is not admitted. In the event that some links on the path become congested and cannot support the maximum delivery speed, the protocol has mechanisms to divert the traffic along other routes. SPEED uses a back-pressure re-routing technique to overcome packet delivery degradation due to congestion. The back-pressure rerouting scheme avoids packet traversal over congested links, thus ensuring that the packet's delivery speed is maintained. Simulation results indicate high packets delivery ratios along with energy efficiency and effective load balancing. One drawback of SPEED is that it does not have a prioritization scheme for the packets. In addition, each forwarding node can only forward the packet at a speed less than or equal to the maximum delivery speed. Even if parts of the network support a higher speed and a packet at some hop in that part requires a higher speed (to make up for congestion delay at previous hops) it cannot be forwarded at that higher speed. In a wireless sensor network, due to the highly dynamic link and route characteristics, per-flow reservation appears to be in general not scalable; hence SPEED likely will not be scalable for large networks.

The Sequential Assignment Routing (SAR) approach, a constant priority approach, suggested by Sohrabi et al. [103] was one of the first approaches in the area of QoS for wireless sensor networks. In this approach, each packet of a given flow/session has a constant priority value and the value remains unchanged while traversing the route to the destination. SAR uses a table driven multi-path approach to find multiple paths that satisfy the QoS and energy conservation requirements. The source node selects a particular route among the multiple available paths for a given admitted flow. The decision is made considering the delay requirements of the flow and the load balancing intentions of the source. The intermediate nodes forward a packet according to its priority. A key advantage of this approach is that it supports multiple priority levels for the sessions. On the other hand, the table driven nature of the algorithm is a major drawback as it requires significant memory at the sensor nodes to store the table and may not be scalable for large networks. In addition, the fact that a packet's priority cannot change, makes it difficult to maintain the guarantee in the event of unforeseen changes in the network.

Felemban et al. [101] presented a differentiated priority packet delivery mechanism called the Multi-path MultiSPEED routing protocol (MMSPEED). MMSPEED provides a probabilistic QoS guarantee by provisioning in both the timeliness and reliability domains. QoS differentiation in reliability is obtained by using multi-path routing with the number of paths dependent on the required degree of reliability for the packet. QoS differentiation in timeliness is obtained by providing multiple network-wide packet delivery speed guarantees. The scheme employs localized geographic forwarding with dynamic compensation to offset inaccuracies in decisions made with only local knowledge. The intermediate nodes have the ability to boost a packet's transmission speed to higher levels if they see that the packet may not meet its delay deadline at the current speed, but the deadline may be met at a higher speed. MMSPEED assumes the use of IEEE 802.11e at the MAC layer with its inherent prioritization mechanism based on the Differentiated Inter-Frame Spacing (DIFS). Each speed value is mapped onto a MAC layer priority class. The Real-Time Communication Architecture (RAP) proposed by Chengyang et al. [100] supports a similar multiple priority scheduling of packets using a velocity monotonic scheduler, which prioritizes the packets and schedules them on the basis of their required speed of transmission. Both the MMSPEED and RAP schemes do not consider the number of hops that the packet has to traverse in deciding the priority. Two packets $P$ and $Q$ that are at the same distance from their corresponding destinations have the same priority. However, if $P$ travels over a larger number of hops than $Q$, then $P$ experiences a larger delay as the priority does not consider the number of hops. Hence, the number of hops is a more realistic measure for priority assignment than the distance (in $\mathrm{m}$ ) between the source and the destination.

The MMSPEED protocol addresses multiple aspects of the QoS issue for multimedia traffic in wireless sensor networks. However, many issues, such as network layer aggregation, managing the energy-delay trade-off, and facilitating param- 
TABLE V

COMPARISON OF QOS SCHEMES AT THE NETWORK LAYER

\begin{tabular}{|c|c|c|}
\hline Scheme & Approach used & Drawbacks \\
\hline \multicolumn{3}{|c|}{ Schemes providing timeliness } \\
\hline SPEED [99] & $\begin{array}{l}\text { Back-pressure re-routing } \\
\text { to control congestion }\end{array}$ & $\begin{array}{l}\text { No priority and delivery guarantees, and } \\
\text { a packet's speed cannot be increased }\end{array}$ \\
\hline SAR [80] & $\begin{array}{l}\text { Multi-path routing for load balancing and } \\
\text { support for multiple priorities }\end{array}$ & $\begin{array}{l}\text { Table driven approach non-scalable and } \\
\text { packet priorities are constant }\end{array}$ \\
\hline EAQos [98] & $\begin{array}{l}\text { Guarantees best effort traffic transmission } \\
\text { and also maximizes real-time traffic throughput }\end{array}$ & $\begin{array}{l}\text { Multiple priorities not supported and multi-path } \\
\text { calculations need complete topology knowledge }\end{array}$ \\
\hline MMSPEED [101] & $\begin{array}{l}\text { Supports multi-path routing and multiple } \\
\text { network-wide packet delivery speeds }\end{array}$ & $\begin{array}{l}\text { Does not handle network layer } \\
\text { aggregation, energy-delay trade-off }\end{array}$ \\
\hline RAP [100] & $\begin{array}{l}\text { Geographic Routing, velocity } \\
\text { monotonic scheduling, and prioritized MAC }\end{array}$ & $\begin{array}{l}\text { Does not include multi-path routing } \\
\text { nor consider queuing delay in calculating } \\
\text { packet priority }\end{array}$ \\
\hline \multicolumn{3}{|c|}{ Schemes providing reliability } \\
\hline ReInForM [102] & $\begin{array}{l}\text { Reliability based on information } \\
\text { in the dynamic packet state }\end{array}$ & $\begin{array}{l}\text { Does not consider delay deadlines of the } \\
\text { packets while choosing the multiple paths. }\end{array}$ \\
\hline MMSPEED [101] & $\begin{array}{l}\text { Packet forwarded with prescribed probabilities } \\
\text { multiple packets sent on multiple paths }\end{array}$ & $\begin{array}{l}\text { Requires substantial state information to be } \\
\text { stored at intermediate SNs }\end{array}$ \\
\hline
\end{tabular}

eters exchange between the application and MAC layer, are largely unaddressed.

\section{B. Reliability}

The common mechanism for providing reliability in wireless sensor networks is multi-path routing. Sending copies of the same data over different paths that are node/edge disjoint increases the probability of at least one of the copies reaching the base station in time and without errors. Both ReInForM [102] and MMSPEED [101] propose approaches based on multi-path routing to provide reliability.

The ReInForM [102] end-to-end reliability mechanism considers the importance of the data in the packet and the channel error conditions to create a differential allocation of network resources. More specifically, based on the importance of the data inside a packet, defined by a Dynamic Packet State (DPS), the packet is assigned a priority level. Each priority level maps to a desired level of reliability. Multiple copies of each packet are sent along multiple paths from source to sink, to ensure that the desired level of reliability is achieved. Each intermediate node on the forward route simply forwards the received packet. If a node is not on the forward route, it makes a decision for forwarding the packet based on the packet's DPS information, in an effort to increase the reliability. This scheme is not designed specifically for real-time or multimedia traffic, so the protocol does not consider the delay deadlines of the packet in selecting the multiple paths. A chosen path may not be able to meet the deadline, however it will still be used. This wastes precious resources of the sensor nodes without improving the system performance.

In MMSPEED [101], based on the importance of the data in the packet, the source node assigns a required reliability probability to each packet. In addition, each node in the network calculates the reliable forwarding probabilities of each of its neighbors to a destination, using the packet loss rate at the MAC layer. Based on the required reliability probability of the packet and the possible reliable forwarding probabilities of its neighbors, the source node sends the packet to a group of neighbors in the direction of the destination. Each intermediate forwarding node uses the same mechanism to forward the packet to one or more of its neighbors. A drawback of this mechanism is that it requires each node to store the reliable forwarding probabilities of its neighbors which need to be updated proactively at different time intervals.

\section{MAC LAYER ReQuirements And Mechanisms For STREAMING APPLICATIONS}

In a multimedia wireless sensor networks, there are two main new performance requirements for the MAC (link) layer in addition to the conventional MAC layer requirements, such as bandwidth provisioning, power awareness, contention resolution abilities, collision avoidance, and interference minimization [104]. The two new performance requirements are: $(i)$ to ensure that the packet latency is optimized to meet the end-to-end delay requirements, and ( $i i)$ to ensure that multiple priorities are provided to packets with varying service requirements. Moreover, MAC schemes for multimedia communications in wireless sensor networks need to be power/energy-aware. While there has been some work on designing energy aware MAC protocols, see e.g., [105], [106], these schemes have not considered real-time scheduling and real-time guarantees for multimedia traffic in combination with energy awareness. Power saving schemes, such as putting the sensor nodes to sleep or using different power levels, may adversely affect the latency and throughput of the multimedia traffic and require further study in the context of multimedia traffic.

In the recent literature, some MAC protocols have been proposed for wireless sensor network that support real-time multimedia communication and we comprehensively survey these protocols in this section. Before we begin our survey, we briefly note that MAC schemes for wireless ad hoc networks have been surveyed in [113] and that selected MAC schemes for wireless sensor networks have been surveyed in [8]. However, these existing surveys do not provide a comprehensive overview of the existing approaches, nor do they give a classification of the schemes. In this survey we introduce a classification of the QoS schemes in the wireless sensor networks based on the underlying mechanism used and discuss how suitable the different categories are for supporting multimedia streaming application traffic in wireless sensor 


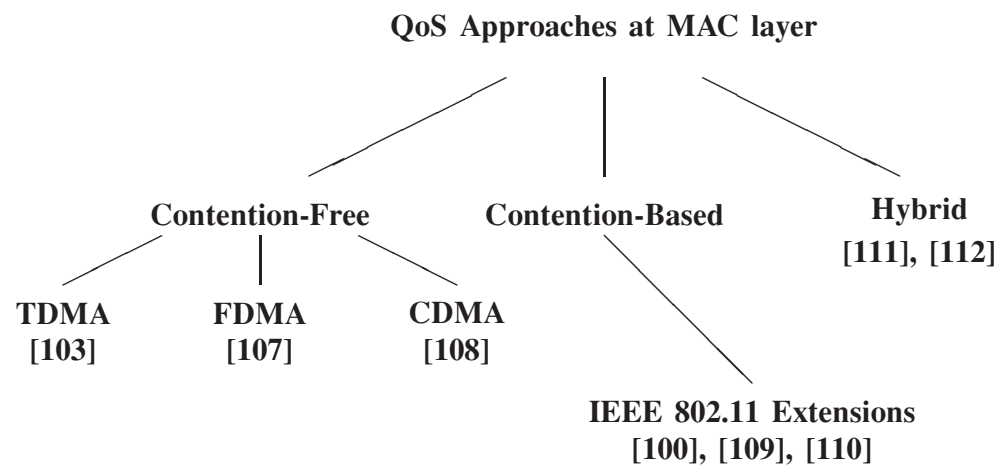

Fig. 7. Classification of MAC Layer QoS solutions

networks. Figure 7 illustrates our classification of the MAC schemes into the categories of contention-free, contentionbased, and hybrid schemes.

\section{A. Contention-Free Schemes}

Contention-free MAC schemes are generally based on TDMA, FDMA, or CDMA [114]. Sohrabi et al. [103] proposed a TDMA based MAC protocol named Self-organizing Medium Access Control for Sensor networks (SMACS). SMACS is a distributed protocol that enables nodes to discover their neighbors and establish transmission/reception schedules for communication, without the help of a global authority. The neighbor discovery and channel assignment phases are combined in SMACS. A communication link consists of a pair of nodes operating at a randomly chosen but fixed frequency (or frequency hopping sequence). Power conservation is achieved by using a random wake-up schedule during the connection phase and by turning the radio off during idle time slots. There are two drawbacks of this mechanism, namely that the number of slots between two neighbors is fixed and that the mechanism needs time synchronization. The use of a fixed number of slots is inflexible, making it difficult to support higher bandwidths. And the TDMA scheme requires the nodes to spend a significant amount of time performing time synchronization in at least the local neighborhood.

Caccamo et al. [107] presented a network architecture with a medium access control protocol that employs Earliest Deadline First (EDF) scheduling [115] for the periodic multimedia traffic to guarantee delay bounds. The network is divided into cells and sensors in adjacent cells use different frequencies based on frequency division multiplexing (FDM), hence resulting in FDMA across adjacent cells without interference. A TDMA-like scheme is created in each cell according to a monotonic ordering obtained by EDF scheduling. Messages are classified as intra-cell (messages exchanged by nodes in a single cell) or inter-cell (message exchanged between nodes in different cells). The nodes in a given cell exchange activity information and maintain common knowledge of the EDF schedule in the cell. Each cell has a router that aids in inter-cell communication. This scheme guarantees delivery, bandwidth, and delay constraints of the admitted real-time traffic. However, it appears that the assumptions in the scheme are not easy to enforce. A cellular network structure and a router in the center of each cell are not consistent with the vision of an infrastructureless network. In addition, the exchange of schedules by the nodes in a cell at periodic intervals may contribute to faster energy depletion.

Liu et al. [108] proposed the use of a CDMA based MAC layer scheme for supporting real-time traffic in wireless sensor networks. The motivation for the CDMA approach is that TDMA based schemes require global synchronization and make bandwidth adjustments difficult. Liu et al. argue that the use of the CDMA scheme provides flexibility in inter-cell bandwidth adjustments, better security, and throughput while allowing independent temporally and spatially overlapping communication. They adopt the same hexagonal cell structure as [107] but instead of using different frequencies, they use different CDMA encoding sequences. As a result a sender in cell labeled ' $i$ ', uses the CDMA code labeled ' $i$ '. Each node has one sender module and six receiver modules functioning at the time of sending, while there are seven receiver modules at the time of listening/receiving. This scheme may result in less inter-channel interference and higher bandwidth utilization than the TDMA and FDMA schemes. However, the use of multiple senders/receivers at a node is expensive and requires specialized sensor hardware.

In essence, the contention-free schemes are promising because they eliminate collisions, increase throughput, reduce delay, and provide real-time guarantees, thus providing good support for multimedia streaming applications. However, on the downside, they are complex, require centralized control, and use multiple channels at the same time, which is usually not supported by current sensor hardware.

\section{B. Contention-Based Schemes}

Several MAC layer algorithms have been proposed for wireless ad hoc networks that are based on contention and carrier sensing, e.g., [100], [105], [110], [116], [117]. Owing to the similarity of the wireless medium, these algorithms may be used in wireless sensor networks as well. IEEE 802.11e, which has provisions for service differentiation at the MAC level [109], is the most widely used basis for designing contention-based schemes. In these schemes, the service differentiation is achieved by changing the duration of the InterFrame Spacing (IFS) and the Contention Window (CW) size based on the priority of the packet. For instance, Veres et al. [110] investigated differentiated services in wireless packet networks using a distributed approach that supports service 
differentiation, radio monitoring, and admission control. Service differentiation is obtained by extending the IEEE 802.11 Distributed Coordination Function (DCF). The algorithm sets the upper bounds for $\mathrm{CW}_{\min }$ and $\mathrm{CW}_{\max }$, the contention window values that define the range of the back-off window, according to the priority level of the packet. For a packet with a higher priority, the $\mathrm{CW}_{\min }$ and $\mathrm{CW}_{\max }$ values are set lower than for packets with a lower priority. This shortens the backoff time for the higher priority packet.

Lu et al. [100] proposed a packet scheduling policy (RAP) that accounts for both distance and time constraints. The MAC layer protocol in this scheme is an extension of IEEE 802.11, which uses priority based values for inter-frame spacing and back-off window values, similar to IEEE 802.11e. Simulation results indicate the suitability of the policy for communication scheduling in sensor networks where the sensor nodes perform real-time monitoring and control. Most of the other schemes based on IEEE 802.11 follow the same principle.

In general, contention based schemes are easy to use, are scalable, and can handle varying traffic volumes unlike the contention-free schemes which required fairly accurate estimates of the traffic volumes. However, unlike contentionfree schemes, the contention based schemes cannot provide real-time guarantees. For these schemes to be successfully applied in the multimedia wireless sensor network domain they need to provide some form of statistical guarantees to the admitted traffic.

\section{Hybrid Schemes}

The hybrid QoS approach at the MAC layer entails the use of concepts from both contention-based and the contentionfree schemes. In a hybrid scheme, the transmission period consists in general of two sub-periods, the reservation (contention) period and the transmission (contention-free) period. During the reservation period the sensor nodes in a neighborhood contend for transmission rights and transmission time in the transmission period, based on the amount of data they have to transmit. Once given access to some slots in the transmission period, the sender and the receiver communicate, see e.g., [111], [112]. We give some insights into the hybrid power-aware reservation based MAC protocol for static ad hoc/sensor networks as proposed by Adamou et al. in [111], which is representative of the hybrid schemes. In [111], the network is partitioned into grids, whereby all the nodes in a grid can communicate with each other. Time is divided into fixed-size frames containing a reservation period and a contention-free period. The nodes in a grid exchange three messages in each reservation period to reserve a slot during which to send/receive data. Once the reservation is completed, data can be sent/received by the nodes in the contentionfree period. The delay bounds of real-time traffic are satisfied if the reservation and the packet transmission are completed before the deadline. The scheme is scalable and can support multiple traffic flows in the network and each node requires only knowledge of its neighborhood. Control packet overhead and packet re-transmissions appear to be minimal in this scheme. The main drawback of this scheme is that the nodes in a neighborhood require synchronization to ensure proper slots reservations in the reservation period. Hence, the hybrid schemes require more interaction between the nodes in comparison to the contention-based schemes.

\section{Summary}

Of the three types of approaches presented in our classification, the hybrid schemes appear to be most suitable for supporting real-time communication in wireless sensor networks. This is because they provide a mechanism to guarantee support for the real-time traffic, while promoting energy efficiency and scalability. The hybrid schemes will likely prevent starvation of best-effort traffic in a real-time traffic dominated scenario, thus improving overall bandwidth utilization.

Despite many QoS aware MAC protocols that have been proposed for wireless ad hoc networks in general and sensor networks in particular, there still exist open problems that need to be solved. No protocol considers the issue of reducing the data redundancy and the energy-delay trade-offs in realtime applications. We believe that an effective MAC protocol should strive for a proper balance between the complexity of service guarantees for multiple service classes, efficient use of available resources, and have the ability to promptly react to failed transmissions. Most of the existing MAC schemes focus on only a subset of QoS features while ignoring other issues, such as end-to-end packet delay in multi-hop networks, channel errors, power control, and heterogeneous nodes. Another open problem is that many wireless sensor networks use topology control and power management algorithms that operate at the MAC layer (or network layer). Power management and topology control aim to make the network sparse, which may adversely affect the throughput and endto-end guarantees in the network. The algorithms proposed need to be re-evaluated in light of the requirements of the multimedia streaming application traffic.

\section{CROSS-LAYER ApProACH TO SUPPORT MUltimedia TRAFFIC}

Supporting multi-priority multimedia traffic consisting of video and audio, along with the standard sensed data traffic is a challenging task. To satisfy the stringent requirements of the real-time traffic, a closer interaction between the layers of the network protocol stack, i.e., cross-layer optimization has been proposed [118]. For cross layer optimization, it is important that the QoS requirements of the application are efficiently and correctly mapped onto the dependent performance parameters at each layer. Cross-layer optimization requires a shift from the modular approach of the stack where each layer performs independently from the other, to a holistic and inter-dependent approach. For instance, the network layer may act as an intermediate between the application and MAC layer for effective mapping of the performance parameters. The network layer has knowledge of the end-to-end characteristics along the route. The MAC layer has knowledge of only the one hop characteristics. The application layer has no information about the network conditions and has only the information about the application. To leverage cross-layer information for meeting application level QoS requirements it becomes essential for all three layers to collaborate, with the network 
TABLE VI

COMPARISON OF SCHEMES AT THE MAC LAYER

\begin{tabular}{|c|c|c|}
\hline Scheme & Approach used & Drawbacks \\
\hline \multicolumn{3}{|c|}{ Contention-Free Schemes } \\
\hline FDMA-based [107] & $\begin{array}{l}\text { Similar to cellular network, inter-cell FDMA, } \\
\text { intra-cell TDMA, EDF scheduling }\end{array}$ & $\begin{array}{l}\text { Needs multiple frequencies, router at the } \\
\text { center of cell, and exchange of schedules }\end{array}$ \\
\hline CDMA-based [108] & $\begin{array}{l}\text { Less inter-channel interference and } \\
\text { more available bandwidth }\end{array}$ & $\begin{array}{l}\text { Requires expensive hardware with } \\
\text { multiple network interfaces at the SNs }\end{array}$ \\
\hline \multicolumn{3}{|c|}{ Contention-based Schemes } \\
\hline $\begin{array}{c}\text { IEEE 802.11 extensions } \\
{[100],[105],[110],[116],[117]}\end{array}$ & $\begin{array}{l}\text { Extensions added to the basic IEEE } 802.11 \text {, } \\
\text { suitable for real-time monitoring and control }\end{array}$ & $\begin{array}{l}\text { Sub-optimal use of bandwidth } \\
\text { because of contention }\end{array}$ \\
\hline \multicolumn{3}{|c|}{ Hybrid Schemes } \\
\hline $\begin{array}{l}\text { Contention phase followed by } \\
\text { contention-free phase [111], [112] }\end{array}$ & $\begin{array}{l}\text { sensor nodes contend for the medium } \\
\text { sensor nodes winning the medium have } \\
\text { contention-free access to medium }\end{array}$ & $\begin{array}{c}\text { May require time synchronization } \\
\text { between SNs, but appears } \\
\text { most suitable }\end{array}$ \\
\hline
\end{tabular}

layer mapping the application level QoS parameters to the MAC layer performance parameters. Despite notable research efforts [118]-[123], the mapping of the QoS parameters across different layers for the purpose of cross-layer optimization still remains elusive.

\section{A. Overall Cross-Layer Optimization Frameworks}

Cross-layer optimization frameworks for wireless sensor networks have been proposed by Schaar and Shankar [118] as well as Goldsmith and Wicker [119]. Figure 8 provides a schematic view of the framework proposed in [118]. As Schaar and Shankar note, it is difficult to achieve a solution to the cross-layer optimization problem represented by the framework because deriving expressions for the queue length, delay, and power consumption in terms of the channel conditions is challenging due to their non-linearity and interdependence characteristics. The algorithms and protocols at the different layers operate on different units of multimedia traffic and depend on different performance variables. Also, each protocol is optimized for the concerned layer, independent of the protocols operating at the other layers. The wireless channel conditions and multimedia characteristics change frequently, requiring parameter adjustments. The cross-layer approach requires formal methods for establishing optimal initialization, grouping of strategies at different stages, and proper ordering for optimization. Different practical considerations, such as buffer sizes, choice of retry limits, and modulation strategies, also need to be considered during cross-layer optimization.

The number of strategies to consider for cross-layer optimization are many and the possible solution space is extensive, hence it is difficult to choose a possible solution from this space. Schaar and Shankar proposed to use iterative optimization or a decision tree approach, wherein a group of strategies are optimized while keeping the others fixed and the process is repeated until convergence. For the optimization of each group of strategies they suggested the use of linear and non-linear programming methods.

Throughout the joint optimization process, the concept of layering can be considered as a means for decomposition of the optimization. The overall communication network is modeled as a generalized network utility maximization problem. Each layer of the stack corresponds to a decomposed sub-problem with the optimization variables acting as the interfaces, coordinating between the sub-problems [120].

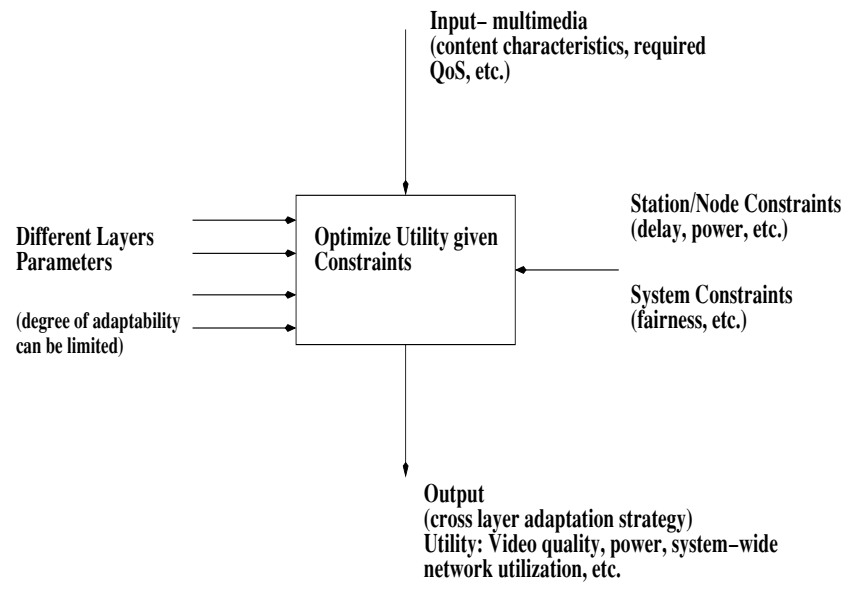

Fig. 8. The conceptual framework for cross-layer optimization proposed by Schaar and Shankar in [118]

\section{B. Specific Cross-Layer Optimization Approaches}

In this section, we review representative cross-layer optimization approaches that jointly consider a specific combination of network layers.

1) Approaches Focused on Minimizing Power Consumption: One of the most extensively studied joint optimization problems is that of power minimization for coding and communication. The work by Eisenberg et al. [124], a representative example of joint power optimization, minimizes the energy required to transmit video while satisfying distortion and delay constraints. The optimization problem jointly takes into account the error resilience and concealment techniques used by the video coder and the transmission power management at the physical layer. The solution to the problem identifies the minimum number of bits and the minimum average power required to transmit a given packet with the acceptable distortion and within the delay bound. The video coding considered is block-based motion-compensated videocoding, such as MPEG or H.26x. However, similar analysis can be applied to other video coding algorithms. From their simulations, the authors conclude that adjusting source coding and physical layer parameters together is a more suitable technique in comparison to adapting each independently.

Power minimizations is also considered by Gastpar and Vetterli [125] who studied joint source-channel optimization and presented a lower bound on the best achievable end-toend distortion as a function of the number of sensors, their 
total transmit power, the number of degrees of freedom of the underlying source process, and the spatio-temporal communication bandwidth. They show that the standard practice of separating source from channel coding could potentially incur an exponential delay and throughput penalty in communication, as a function of the number of sensors, hence, such code designs are not scalable.

Yuan and Yu [126] consider a joint optimization of source coding and power allocation for the CEO (Central Estimation Officer) problem in a sensor network. They minimized the overall distortion and power consumption. They employed distributed source coding, which is well suited for wireless sensor networks because it trades off the transmission rate of one sensor for that of another sensor in the neighborhood. The authors proposed a source coding formulation that characterized this coupled relationship between sensor nodes in a neighborhood and they minimize rate distortion and power via convex programming. Yu and Yuan extended their work in [126] to include routing. They proposed an optimization framework that jointly solves source coding, routing, and power allocation. The distributed source coding problem at the application layer and the power allocation problem at the physical layer are in general, non-convex optimization problems. However, the authors suggested that these can be turned into convex optimization problems if time-division and frequencydivision multiplexing are used. They also note that the overall optimization problem decomposes into the subproblems for each layer, with the Lagrangian dual variables serving as coordinating cross-layer interfaces. The simulations-run on a very small network-indicated fast convergence of the dual variables, with the global optimum for the joint optimization reached at the convergence point.

2) Approaches Focused on Maximizing Throughput: There are many works that attempt to perform joint optimization of routing and scheduling as well as the link and physical layer functionalities (or a combination thereof) to obtain the maximal throughput with the best possible energy-efficiency. For instance, Cui et al. [127] study joint routing, scheduling, and link adaptation strategies to maximize network lifetime. A variable length TDMA scheme is proposed to minimize energy consumption in the network by preventing contention-based collisions. The slot length for the scheme is optimally assigned according to the routing requirement. The results show that the energy consumption in multi-hop routing is dominated by transmission energy whereas, single-hop transmission should consider circuit processing energy as an equally contributing factor for energy consumption. In [128], an extension of [127], Cui et al. proposed an iterative algorithm that performs adaptive link scheduling and computes optimal link rates and transmission powers for a fixed link schedule, given that the TDMA-based link schedules are non-orthogonal. Similar combinations of joint power, scheduling, routing, and physical layer optimizations were studied in [121]-[123], [129].

\section{Discussion}

All of the reviewed approaches have similar limitations. First, most of the approaches are centralized or only partially distributed, limiting their scalability. There is need for efficient distributed schemes that are scalable, while at the same time requiring minimal energy and message exchanges. Second, very few schemes have been implemented in real sensor networks, and those that have been implemented, have been tested only for small networks. Most of the works rely on numerical validations; however, whether the numerical results translate into reality is a subject of study. We note here that the lack of real-world implementations is attributable to the complexity of such implementations and to the many unrelated issues that need to be addressed for experimental implementation. Third, the networks studied in the numerical analysis are small. It is well known that optimization schemes scale very slowly for large networks, hence, the efficiency of the reviewed schemes needs to be gauged for large network sizes. We conclude from our survey of cross-layer approaches that joint optimizations are a promising approach to attain optimality in performance across the protocol stack, however, their feasibility needs to be studied in greater detail. There is need for research that performs joint optimization in a distributed manner and examines the performance penalty associated with state maintenance and the exchange of state information in the distributed protocols for large scale wireless sensor networks.

A simple cross-layer approach that we believe could serve as a starting point for comprehensive evaluations is one that uses the best protocol/mechanism at each layer of the stack, independent of those used in the other layers, that is, using the most optimized protocol at each layer. In this survey, we have looked at various approaches and protocols that exist at each layer of the network stack for supporting MSAs. Each of the protocols attempts to provide an optimum solution at its corresponding layer. Thus, each of these protocols is inherently optimized, independent of the other layers and their interactions. A combination of protocols - each optimized at its corresponding layer-appears to be sub-optimal in the unified sense, however, it could serve as a good benchmark. Also, this approach would be better than naively choosing any protocol at each layer of the stack without considering its optimality given the operating conditions.

We proceed to examine the approach of selecting the optimal protocol for each individual layer in more detail. At the application layer, distributed source coding and multilayer error-resilient coding stand out as preferable schemes with applicability in a wide range of scenarios. Distributed coding is well suited for a wireless sensor network where the nodes are densely deployed. In a dense network, the redundancy due to significant overlap can be exploited with distributed coding. In addition, multi-layer coding is preferable for a wireless sensor network operating in a highly lossy and dynamic environment. Also, in a wireless sensor network where the bandwidth available to nodes varies over a wide range, the multi-layer scheme may be able to perform better as the coding can adapt to the available bandwidth and be error-resilient.

At the transport layer, we did not find any protocols that are suitable for multimedia streaming traffic. The ESRT and STCP protocols come close to a solution as they handle both congestion control and reliable transport. However, both require major enhancements for being able to seamlessly 
support multimedia streaming traffic. Hence, we consider an ESRT/STCP based scheme with enhancements that address outstanding issues, such as support for multi-path routing, data segmentation and assembly, and faster and more scalable congestion control, as the transport protocol of choice.

At the network layer, the differentiated priority scheme with adjustable priority for each packet seems to be best suited for the dynamic wireless environment. This is because it allows a packet's priority to be enhanced if it is in danger of not meeting its deadline at the current priority. This mechanism reduces the number of real-time packets that fail to meet their deadlines. Multi-path routing appears to be an essential feature for supporting multimedia streaming applications as it improves the chance of data delivery within the stipulated deadline.

At the MAC layer, we believe that the hybrid schemes stand out as the preferred solution for supporting MSAs. The hybrid schemes combine the best of the contentionfree and contention-based schemes to provide bandwidth and delay guarantees to the admitted traffic while operating in a distributed manner. The contention-free scheme is useful in a special type of wireless sensor network with knowledge of the nodes positions and the bandwidth requirements of the applications. In this scenario, contention-free schemes give the best result. However, application scenarios of such wireless sensor networks are limited.

Using the different options at each layer we propose the following cross-layer solutions:

- In a densely deployed network, employ distributed coding at the application layer and either STCP/ESRT at the transport layer, along with the differentiated priority scheme with multi-path routing (if necessary) at the network layer, and a hybrid scheme at the MAC layer.

- In a scattered to medium density wireless sensor network with little overlap between the data of the SNs, employ the multi-layered scheme at the application layer, the STCP scheme at the transport layer, and the differentiated scheme at the network layer. The MAC layer approach could be a hybrid scheme or a contention-free scheme if the required position and bandwidth requirements knowledge exists.

- In a wireless sensor network with a noisy medium, the multi-layer scheme seems to be the better solution even if the network is dense. This is because the noise may corrupt the message exchanges in a distributed coding scheme, resulting in fast depletion of energy in the sensor nodes due to frequent re-transmissions. Also, multi-path routing is essential in this case to reduce the risk of quality degradation resulting from packet loss.

The cross-layer approach suggested by Schaar and Shankar, of selective optimization of the protocols in a group while keeping others constant and then similarly optimizing the remaining groups one at a time until convergence, is another possible mechanism for cross-layer solutions. Again, the approach may not be able to obtain a globally optimal solution, however, a study of its deviation from optimal performance would be interesting. The selective optimization approach of Schaar and Shankar can also be envisioned as a tuning mechanism, where the protocol in one layer is tuned on the basis of the protocols of the upper layers to provide the best integration. A similar approach can be applied in a bottomup manner, wherein the protocols of the upper layers are tuned/chosen on the basis of the performance of the optimized protocols for the lower layers. The comparative performance analysis of the top-down and bottom-up approaches would be an interesting study.

Cross-layers solutions to the issues in WSNs, especially those pertaining to supporting multimedia applications are in a fairly early phase of development. However recent works in this area have underlined the possible advantages of using a cross-layer approach for maximizing the resource utilization and solving the interaction problems in wireless networks. We believe that more attention needs to be focused on crosslayer solutions including joint optimization to improve the performance of multimedia streaming applications in WSNs.

\section{CONCLUSION}

This survey has explored real-time multimedia streaming in wireless sensor networks. We have presented the requirements of the real-time multimedia streaming traffic that need to be met by the application, transport, network, and the MAC layer. We have surveyed the schemes proposed for each layer, and have introduced classifications for these schemes on the basis of the mechanisms they use. The viability of the cross-layer approach for providing the QoS requirements of the real-time multimedia application has been explored. We have outlined a few directions for the development of cross-layer solutions that can be used for given typical wireless sensor networks and wireless communications conditions. We also presented in this survey the open problems that have not been addressed by the existing schemes, but we believe need to be addressed for efficient support for multimedia streaming applications.

This survey suggests directions for further research, namely investigating in detail the cross-layer solutions that we outlined and exploring new optimization approaches. Overall, comprehensive quantitative comparisons of the proposed solutions for the individual layers as well as cross-layer solutions are an important direction for future research. In addition, there exists a possible scope for further research at each layer of the stack. At the application layer, there is a need for schemes that exploit the co-operative nature of the sensor nodes while at the same time being adaptive and resilient. The distributed and multi-layer coding schemes as well as video fusion are steps in this direction. However, a scheme that can combine the positive traits of distributed and multilayer coding while still being efficient is desirable. Testbed or real-world implementation of distributed source coding in wireless sensor networks is an important next step in research. Such implementations would open up new issues, such as identification of the correlation models which are application specific and identification of the parameters for performance tuning.

At the transport layer, the existing protocols have not been designed to support multimedia streaming traffic. There is a significant scope for research in this area, which should primarily focus on distributed protocols for providing the requirements of the multimedia streaming applications. 
At the network layer, not much work has been done on the constant priority approaches, as may be observed from the presented classification. A future research direction is to evaluate the efficacy of these different approaches and to identify the best approach. In addition, the routing protocols have to be extended to address the energy-delay trade-off, and be equipped to facilitate the exchange of performance parameters between the application and the MAC layer. At the network layer, simple aggregation algorithms for real-time data are desirable to help wireless sensor networks support multimedia applications better.

At the MAC layer, not many schemes have been proposed that use the contention-free and hybrid mechanisms. We believe, the mechanisms identified in the classification need to be thoroughly evaluated to identify mechanisms most suited for a wireless sensor network environment. The IEEE 802.11e schemes have been found to be useful in the mobile ad hoc environment because of their distributed nature and independence from node synchronization. These contentionbased schemes may be put to a wider use in the wireless sensor network environment by improving the statistical guarantees provided to real-time traffic. As previously suggested, at the MAC layer, the issues of energy-delay trade-off, reduction in data redundancy, and handling of channel error need to be addressed. Another possibly essential direction at the MAC layer is the study of power management and topology control schemes in light of the requirements of the multimedia streaming applications.

Multimedia streaming applications will likely become very popular in wireless sensor networks in the near future. With the focus on miniaturization and given the breakthroughs in embedded technology, sensor nodes will keep getting smaller and more powerful. In light of these improvement in abilities, we believe that new sensor node protocols have to be developed that leverage cross-layer information and understand the requirements of real-time multimedia applications, while being aware of the limitations of the wireless sensor networks. The cross-layer approach appears to be a promising direction of research for improving the feasibility of multimedia streaming applications in wireless sensor networks.

\section{ACKNOWLEDGMENT}

We are grateful to the anonymous reviewers for their thoughtful and detailed feedback which has helped to greatly improve this survey.

\section{REFERENCES}

[1] I. Akyildiz, W. Su, Y. Sankarasubramaniam, and E. Cayirci. Wireless sensor networks: A survey. Computer Networks, 38(4):393-422, December 2002.

[2] C. Chiasserini and E. Magli. Energy consumption and image quality in wireless video-surveillance networks. In Proc. 13th IEEE International Symposium on Personal, Indoor and Mobile Radio Communications (PIMRC), pages 2357-2361, Lisboa, Portugal, September 2002.

[3] W.-C. Feng, E. Kaiser, W. C. Feng, and M. LeBaillif. Panoptes: scalable low-power video sensor networking technologies. ACM Trans. Multimedia Computing, Communications, and Applications, 1(2):151167, May 2005.

[4] P. Kulkarni, D. Ganesan, P. Shenoy, and Q. Lu. SensEye: a multi-tier camera sensor network. In Proc. 13th Annual ACM Int. Conference on Multimedia, pages 229-238, November 2005.
[5] H. Wu and A. Abouzeid. Energy efficient distributed JPEG 2000 image compression in multihop wireless networks. In Proc. 4th Workshop on Applications and Services in Wireless Networks (ASWN), pages 152160, August 2004.

[6] K. Obraczka, R. Manduchi, and J.J. Garcia-Luna-Aveces. Managing the information flow in visual sensor networks. In Proc. 5th International Symposium on Wireless Personal Multimedia Communications, volume 3, pages 1177-1181, October 2002.

[7] D. Chen and P. K. Varshney. QoS support in wireless sensor networks: A survey. In Proc. International Conference on Wireless Networks $(I C W N)$, pages 21-24, Las Vegas, NV, June 2004.

[8] M. Younis, K. Akkaya, M. Eltowiessy, and A. Wadaa. On handling QoS traffic in wireless sensor networks. In Proc. 37th Hawaii International Conference on System Sciences (HICSS), pages 90292-90302, January 2004.

[9] E. Gurses and O. Akan. Multimedia communication in wireless sensor networks. Proc. Annals of Telecommunications, 60(7-8):799$827,2005$.

[10] Intel Strong Arm 1100 RISC Embedded Microprocessor. URL: http://www.intel.com/design/strong/datashts/278241.htm.

[11] Stargate embedded sensor. URL: http://www.xbow.com/products/ productsdetails.aspx.

[12] Samsung S3C44B0X RISC Embedded Microprocessor. URL: http://www.samsung.com/products/semiconductor/ mobilesolutions/ mobileassp/mobilecomputing/s3c44b0/s3c44b0.htm.

[13] A. Skordas, C. Chirstopoulos, and T. Ebrahimi. The JPEG 2000 still image compression standard. IEEE Signal Processing Mag., 18(5):3658, September 2001.

[14] W. Feng, B. Code, E. Kaiser, M. Shea, and W. Feng. Panoptes: Scalable low-power video sensor networking technologies. In Proc. ACM Int. Conference on Multimedia, pages 90-91, Berkeley, CA, November 2003.

[15] Z. He, Y. Liang, L. Chen, I. Ahmad, and D. Wu. Power-rate-distortion analysis for wireless video communication under energy constraints. IEEE Trans. Circuits Syst. Video Technol., 15(5):645-658, May 2005.

[16] Z. He and $\mathrm{D}$. Wu. Resource allocation and performance analysis of wireless video sensors. IEEE Trans. Circuits Syst. Video Technol., 16(5):590-500, May 2006.

[17] J. Zhang, G. Zhou, S. Son, and J. Stankovic. Ears on the ground: An acoustic streaming service in wireless sensor networks. In Proc. ACM/IEEE Information Processing in Sensor Networks, Nashville, TN, April 2006.

[18] http://www.xbow.com/.

[19] T. Berger, Z. Zhang, and H. Viswanathan. The CEO problem. IEEE Trans. Inform. Theory, 42(3):887-902, May 1996.

[20] D. Slepian and J. Wolf. Noiseless coding of correlated information sources. IEEE Trans. Inform. Theory, 19(4):471-480, July 1973.

[21] A. Wyner. Recent results in the Shannon theory. IEEE Trans. Inform. Theory, 20(1):2-10, January 1974.

[22] S. Pradhan and K. Ramachandran. Distributed source coding using syndromes (DISCUS): Design and construction. IEEE Trans. Inform. Theory, 49(3):626-643, March 2003.

[23] J. Chou, S. Pradhan, and K. Ramchandran. Turbo and trellis-based constructions for source coding with side information. In Proc. Data Compression Conference (DCC), pages 33-42, March 2003.

[24] A. Liveris, Z. Xiong, and C. Georghiades. Nested convolution/turbo codes for the binary Wyner-Ziv problem. In Proc. IEEE International Conference on Image Processing (ICIP), pages 601-604, September 2003.

[25] A. Liveris, Z. Xiong, and C. Georghiades. Compression of binary sources with side information at the decoder using LDPC codes. IEEE Commun. Lett., 6(10):440-442, October 2002.

[26] A. Wyner and J. Ziv. The rate-distortion function for source coding with side information at the decoder. IEEE Trans. Inform. Theory, 22(1):1-10, January 1976.

[27] R. Wagner, R. Nowak, and R. Baraniuk. Distributed image compression for sensor networks using correspondence analysis and superresolution. In Proc. IEEE International Conference on Image Processing (ICIP), volume 1, pages 597-600, September 2003.

[28] N. Boulgouris and M. Strintzis. A family of wavelet-based stereo image coders. IEEE Trans. Circuits Syst. Video Technol., 12(10):898203, October 2002.

[29] S. Park, M. Park, and M. Kang. Super-resolution image reconstruction: A technical overview. IEEE Signal Processing Mag., 20(3):21-36, May 2003.

[30] B. Song, O. Bursalioglu, A. Roy-Chowdhury, and E. Tuncel. Towards a distributed video compression algorithm. Site: http://www.dvsp.ee.ucr.edu/, University of California, Riverside. 
[31] A. Majumdar and K. Ramchandran. PRISM: An error-resilient video coding paradigm for wireless networks. In Proc. of First International Conference on Broadband Networks (BROADNETS'04), pages 478485, October 2004.

[32] T. Chou, D. Petrović, and K. Ramachandran. A distributed and adaptive signal processing approach to reducing energy consumption in sensor networks. In Proc. IEEE INFOCOM, pages 1054-1062, April 2003.

[33] B. Girod, A. Aaron, S. Raneand, and D. Rebollo-Monedero. Distributed source coding. Proc. IEEE, 93(1):71-83, January 2005.

[34] R. Puri, A. Majumdar, P. Ishwar, and K. Ramchandran. Distributed video coding in wireless sensor networks. IEEE Signal Processing Mag., 23(4):94-106, July 2006.

[35] Z. Xiong, A. Liveris, and S. Cheng. Distributed source coding for sensor networks. IEEE Signal Processing Mag., 21(5):80-94, September 2004.

[36] M. Gerla and K. Xu. Multimedia streaming in large-scale sensor networks with mobile swarms. SIGMOD Rec., 32(4):72-76, December 2003.

[37] E. Magli, M. Mancin, and L. Merello. Low complexity video compression for wireless sensor networks. In Proc. 2003 International Conference on Multimedia and Expo, pages 585-588, July 2003.

[38] G. Pekhteryev, Z. Sahinoglu, P. Orlik, and G. Bhatti. Image transmission over IEEE 802.15.4 and zigbee networks. In Proc. IEEE International Symposium on Circuits and Systems (ISCAS), pages 2326, May 2005.

[39] M. Elmusrati, R. Jantti, and H. Koivo. Distributed sensor network data fusion using image processing. In Proc. Systems Communications, pages 383-388, August 2005.

[40] Z. He and D. Wu. Accumulative visual information in wireless video sensor network: definition and analysis. In Proc. IEEE ICC, pages 1205-1208, May 2005.

[41] H. Ma and Y. Liu. Correlation based video processing in video sensor networks. In Proc. IEEE Int. Conference on Wireless Networks, Communications and Mobile Computing, pages 987-992, June 2005.

[42] F. Zhai, Y. Eisenberg, T. Pappas, R. Berry, and A. Katsaggelos. An integrated joint source-channel coding framework for video transmission over packet lossy networks. In Proc. of IEEE International Conference on Image Processing (ICIP), pages 24-27, October 2004.

[43] C. Leung and A. Lam. Forward error correction for an ARQ scheme. IEEE Trans. Commun., 29(10):1514-1519, October 1981.

[44] E. Martinian and C.-E. Sundberg. Burst erasure correction codes with low decoding delay. IEEE Trans. Inform. Theory, 50(10):2494-2502, October 2004

[45] B. Krasic, J. Walpole, and W. Feng. Quality-adaptive media streaming by priority drop. In Proc. ACM NOSSDAV, pages 112-121, June 2003.

[46] M. Wu and C. Chen. Multiple bitstream image transmission over wireless sensor networks. In Proc. IEEE Sensors, volume 2, pages 727-731, October 2003.

[47] A. Said and W. Pearlman. A new, fast, and efficient image codec based on set partitioning in hierarchical trees. IEEE Trans. Circuits Syst. Video Technol., 6(3):243-250, June 1996.

[48] W. Yu, Z. Sahinoglu, and A. Vetro. Energy efficient JPEG 2000 image transmission over wireless sensor networks. In Proc. Global Telecommunications Conference (GLOBECOM), pages 2738-2743, December 2004

[49] H. Wu and A. Abouzeid. Power aware image transmission in energy constrained wireless networks. In Proc. Ninth International Symposium on Computers and Communications, pages 202-207, July 2004.

[50] W. Heinzelman, A. Chandrakasan, and H. Balakrishnan. Energyefficient communication protocol for wireless microsensor networks. In Proc. Hawaii International Conference on System Sciences, (HICSS), January 2000.

[51] N. Gogate, D-M Chung, S. Panwar, and Y. Wang. Supporting image and video applications in a multihop radio environment using path diversity and multiple description coding. IEEE Trans. Circuits Syst. Video Technol., 12(9):777-792, September 2002.

[52] Y. Wang, M. Orchard V. Vaishampayan, and A. Reibman. Multiple description coding using pairwise correlating transforms. IEEE Trans. Image Processing, 10(3):351-366, March 2001.

[53] J. Apostolopoulos. Reliable video communication over lossy packet networks using multiple state encoding and path diversity. In Proc. of SPIE Visual Communications and Image Processing, pages 392-409, January 2001.

[54] J. Apostolopoulos and S. Wee. Unbalanced multiple description video communication using path diversity. In Proc. IEEE International Conference on Image Processing, pages 966-969, October 2001.

[55] E. Baccaglini, G. Barrenetxea, and B. Beferull-Lozano. Performance of multiple description coding in sensor networks with finite buffers.
In Proc. of IEEE International Conference on Multimedia and Expo (ICME), pages 1460-1463, July 2005.

[56] W. Tan and A. Zakhor. Real-time Internet video using error resilient scalable compression and TCP-friendly transport protocol. IEEE Trans. Multimedia, 1(2):172-186, June 1999.

[57] W. Du, J. Deng, Y. Han, and P. Varshney. A pairwise key predistribution scheme for wireless sensor networks. In Proc. 10th ACM Conference on Computer and Communications Security, pages 42-51, Washington D.C., October 2003.

[58] M. Eltoweissy, M. Moharrum, and R. Mukkamala. Dynamic key management in sensor networks. IEEE Commun. Mag., 44(4):122130, April 2006

[59] L. Eschenauer and V. Gligor. A key-management scheme for distributed sensor networks. In Proc. 9th ACM Conference on Computer and Communications Security, pages 41-47, Washington, D.C., November 2002.

[60] D. Liu, P. Ning, and W. Du. Group-based key pre-distribution in wireless sensor networks. In Proc. 4th ACM Workshop on Wireless security (WiSe'05), pages 11-20, September 2005.

[61] M. Younis, K. Ghumman, and M. Eltoweissy. Location-aware combinatorial key management scheme for clustered sensor networks. IEEE Trans. Parallel Distrib. Syst., 17(8):865-882, August 2006.

[62] S. Zhu, S. Setia, and S. Jajodia. Leap: efficient security mechanisms for large-scale distributed sensor networks. In Proc. 10th ACM Conference on Computer and Communications Security, pages 62-72, Washington D.C., October 2003.

[63] J. Black, S. Halevi, H. Krwaczyk, T. Krovetz, and P. Rogaway. UMAC: Fast and secure message authentication. In Proc. of 19th Annual International Cryptology Conference (CRYPTO), pages 216-245, Santa Barbara, CA, August 1999.

[64] M. Bellare, R. Canetti, and H. Krawczyk. Keying hash functions for message authentication. In Proc. of 16th Annual International Cryptology Conference (CRYPTO), pages 1-15, August 1996.

[65] C. Karlof, N Sastry, and D. Wagner. Tinysec: A link layer security architecture for wireless sensor networks. In Proc. 2nd ACM International Conference on Embedded Networked Sensor Systems (SenSys), pages 162-175, New York, NY, 2004.

[66] C.-C. Chang, M.-S. Hwang, and T.-S. Chen. A new encryption algorithm for image cryptosystems. J. Systems and Software, 58(2):8391, September 2001.

[67] N. Bourbakis and C. Alexopoulos. Picture data encryption using SCAN patterns. Pattern Recognition, 25(6):567-581, 1992.

[68] P. Refregier and B. Javidi. Optical image encryption based on input plane and fourier plane random encoding. Optics Letters, 20(7):767769, April 1995.

[69] J.-C. Yen and J.-I. Guo. A chaotic neural network for signal encryption/decryption and its VLSI architecture. In Proc. 10th VLSI Design/CAD Symposium, pages 319-322, 1999.

[70] H. Chang and J. Liou. An image encryption scheme based on quadtree compression scheme. In Proc. International Computer Symposium, pages 230-237, December 1994.

[71] H.-C. Chen, J.-I. Guo, and L.-C. Yen. Design and realization of a new signal security system for multimedia data transmission. EURASIP J. Applied Signal Processing, 2003(13):1291-1305, 2003.

[72] C. Wang, M. Daneshmand, B. Li, and K. Sohraby. A survey of transport protocols for wireless sensor networks. IEEE Network, 20(3):34-40, March 2006

[73] Stream Control Transmission Protocol. www.sctp.org.

[74] RTP: A Transport Protocol for Real-Time Applications. http://www.ietf.org/rfc/rfc3350.txt.

[75] T. Braun, T. Voigt, and A. Dunkels. Energy-efficient TCP operation in wireless sensor networks. PIK Journal, Special Issue on Sensor Networks, 28(2), 2005.

[76] Z. Fu, X. Meng, and S. Lu. A transport protocol for supporting multimedia streaming in mobile ad hoc networks. IEEE J. Select. Areas Commun., 21(10):1615-1626, December 2004

[77] J. Liu and S. Singh. ATCP: TCP for mobile ad hoc networks. IEEE J. Select. Areas Commun., 19(7):1300-1315, July 2001.

[78] K. Sundaresan, V. Anantharaman, H-Y. Hsieh, and R. Sivakumar. ATP: A reliable transport protocol for ad-hoc networks. In Proc. 4th ACM Int. Symposium on Mobile Ad Hoc Networking \& Computing (MobiHoc), pages 64-75, Annapolis, MD, June 2003

[79] B. Hull, K. Jamieson, and H. Balakrishnan. Mitigating congestion in wireless sensor networks. In Proc. ACM SenSys, pages 134-147, November 2004.

[80] C-Y. Wan, S. Eisenman, and A. Campbell. CODA: Congestion detection and avoidance in sensor networks. In Proc. ACM SenSys, pages 266-279, November 2003. 
[81] C. T. Ee and R. Bajcsy. Congestion control and fairness for many-toone routing in sensor networks. In Proc. ACM Sensys, pages 148-161, Baltimore, MD, November 2004.

[82] C. Wang, K. Sohraby, V. Lawrence, and B. Li. Priority-based congestion control in wireless sensor networks. In Proc. IEEE International Conference on Sensor Networks, Ubiquitous, and Trustworthy Computing (SUTC), pages 22-31, June 2006.

[83] C-Y. Wan, S. Eisenman, A. Campbell, and J. Crowcroft. Siphon: Overload traffic management using multi-radio virtual sinks in sensor networks. In Proc. ACM SenSys, pages 116-129, November 2005.

[84] H. Zhang, A. Arora, Y. Choi, and M. Gouda. Reliable bursty convergecast in wireless sensor networks. In Proc. ACM MobiHoc, pages 266-276, May 2005.

[85] F. Stann and J. Heidemann. RMST: Reliable data transport in sensor networks. In Proc. First International Workshop on Sensor Net Protocols and Applications, pages 102-112, May 2003.

[86] Y. Iyer, S. Gandham, and S. Venkatesan. STCP: A generic transport protocol for wireless sensor networks. In Proc. IEEE International Conference on Computer Communications and Networks (ICCCN), pages $17-19$, October 2005.

[87] Y. Sankarasubramaniam, Ö. Akan, and I. Akyildiz. ESRT: Eventto-sink reliable transport in wireless sensor networks. In Proc. 4th ACM Int. Symposium on Mobile Ad Hoc Networking \& Computing (MobiHoc), pages 177-188, Annapolis, MD, June 2003.

[88] P. Levis, N. Patel, D. Culler, and S. Shenker. Trickle: A self-regulating algorithm for code propagation and maintenance in wireless sensor networks. In Proc. First USENIX/ACM Symposium on Network Systems Design and Implementation (NSDI), March 2004.

[89] C.-Y. Wan, A. Campbell, and L. Krishnamurthy. PSFQ: A reliable transport protocol for wireless sensor networks. In Proc. ACM International Workshop on Wireless Sensor Networks and Applications, pages 1-11, September 2002.

[90] S-J. Park, R. Vedantham, R. Sivakumar, and I. Akyildiz. A scalable approach for reliable downstream data delivery in wireless sensor networks. In Proc. 5th ACM Int. Symposium on Mobile Ad Hoc Networking and Computing (MobiHoc), pages 78-89, May 2004.

[91] C. Intanagonwiwat, R. Govindan, D. Estrin, J. Heidemann, and F. Silva. Directed diffusion for wireless sensor networking. IEEE/ACM Trans. Networking, 11(1):2-16, February 2003.

[92] A. Tanenbaum. Computer Networks. Prentice Hall, New York, NY, 3 edition, 2000

[93] S. Floyd and V. Jacobson. Random early detection gateways for congestion avoidance. IEEE/ACM Transactions on Networking, 1(4):397413, August 1993.

[94] S. Chen and K. Nahrstedt. Distributed quality of service routing in ad-hoc networks. IEEE J. Select. Areas Commun., 17(8):1488-1505, August 1999.

[95] C. Lin. On demand QoS routing in multihop mobile networks. In Proc. IEEE INFOCOM, volume 3, pages 1735-1744, April 2001.

[96] R. Sivakumar, P. Sinha, and V. Bharghavan. CEDAR: A core extraction distributed ad hoc routing. IEEE J. Select. Areas Commun., 17(8):1454-1465, August 1999.

[97] C. Zhu and M. Orson. QoS routing for mobile ad hoc networks. In Proc. IEEE INFOCOM, pages 958-967, June 2002.

[98] K. Akkaya and M. Younis. An energy-aware QoS routing protocol for wireless sensor network. In Proc. Workshops in the 23rd International Conference on Distributed Computing Systems, pages 710-715, May 2003.

[99] T. He, J. Stankovic, C. Lu, and T. Abdelzaher. SPEED: A stateless protocol for real-time communication in sensor networks. In Proc. 23rd International Conference on Distributed Computing Systems, pages 4655, May 2003.

[100] C. Lu, B. Blum, T. Abdelzaher, J. Stankovic, and H Tian. RAP: A real-time communication architecture for large-scale wireless sensor networks. In Proc. IEEE Real-time Systems Symposium (RTSS), pages 55-66, December 2001.

[101] E. Felemban, C-G. Lee, E. Ekici, R. Boder, and S. Vural. Probabilistic QoS guarantee in reliability and timeliness domains in wireless sensor networks. In Proc. IEEE INFOCOM, pages 2646-2657, March 2005.

[102] B. Deb, S. Bhatnagar, and B. Nath. ReInForM: Reliable information forwarding using multiple paths in sensor networks. In Proc. 28th Annual IEEE International Conference on Local Computer Networks, pages 406-415, Bonn, Germany, October 2003.

[103] K. Sohrabi, J. Gao, V. Allawadhi, and G. Pottie. Protocols for selforganization of a wireless sensor network. IEEE Pers.l Commun., 7(5):16-27, October 2000.
[104] J. Stankovic, T. Abdelzaher, C. Lu, L. Sha, and J. Hou. Real-time communication and coordination in embedded sensor networks. Proc. IEEE, 91(7):1002-1022, July 2003.

[105] Y. Wei, J. Heidemann, and D. Estrin. An energy-efficient MAC protocol for wireless sensor networks. In Proc. IEEE INFOCOM, pages 1567-1576, June 2002.

[106] A. Woo and D. Culler. A transmission control scheme for media access in sensor networks. In Proc. 7th ACM Annual Int. Conference on Mobile Computing and Networking (MobiCom), pages 221-235, Rome, Italy, July 2001.

[107] M. Caccamo, L. Zhang, S. Lui, and G. Buttazzo. An implicit prioritized access protocol for wireless sensor networks. In Proc. 23rd IEEE RealTime Systems Symposium (RTSS), pages 39-48, December 2002.

[108] X. Liu, Q. Wang, L. Sha, and W. He. Optimal QoS sampling frequency assignment for real-time wireless sensor networks. In Proc. 24th IEEE Real-Time Systems Symposium (RTSS), pages 308-319, December 2003.

[109] S. Choi, J. del Prado, S. Nandgopalan, and S. Mangold. IEEE 802.11e contention-based channel access (EDCF) performance evaluation. In Proc. IEEE International Conference on Communications (ICC), pages 1151-1156, Anchorage, Alaska, May 2003.

[110] A. Veres, A. Campbell, M. Barry, and S Li-Hsiang. Supporting service differentiation in wireless packet networks using distributed control. IEEE J. Select. Areas Commun., 19(10):2081-2093, October 2001.

[111] M. Adamou, I. Lee, and I. Shin. An energy efficient real-time medium access control protocol for wireless ad-hoc networks. In Work in Progress Session of Eighth IEEE Real-Time and Embedded Technology and Applications Symposium, September 2002.

[112] V. Rajendran, K. Obraczka, and J. J. Garcia-Luna-Aceves. Energyefficient collision-free medium access control for wireless sensor networks. In Proc. 1st Int. Conference on Embedded Networked Sensor Systems (SenSys), pages 181-192, Los Angeles, CA, November 2003.

[113] S. Kumar, V. Raghavan, and J. Deng. Medium access control protocols for ad hoc wireless networks: a survey. Ad Hoc Networks, 4(3):326358, May 2006.

[114] P. Guangyu and C. Chien. Low power TDMA in large wireless sensor networks. In Proc. Military Communications Conference (MILCOM), volume 1, pages 347-351, October 2001.

[115] C. Liu and J. Layland. Scheduling algorithms for multiprogramming in a hard-real-time environment. Journal of ACM, 20(1):46-61, January, 1973.

[116] T. Dam and K. Langendoen. An adaptive energy-efficient mac protocol for wireless sensor networks. In Proc. 1 st Int. Conference on Embedded Networked Sensor Systems (SenSys), pages 171-180, Los Angeles, CA, November 2003.

[117] X. Yang and N. Vaidya. Priority scheduling in wireless ad hoc networks. In Proc. 3rd ACM Int. Symposium on Mobile Ad Hoc Networking \& Computing (MobiHoc), pages 71-79, Lausanne, Switzerland, June 2002.

[118] M. Van der Schaar and S. Shankar. Cross-layer wireless multimedia transmission: Challenges, principles, and new paradigms. IEEE Wireless Commun. Mag., 12(4):50-58, August 2005.

[119] A. Goldsmith and S. Wicker. Design challenges for energy constrained ad hoc wireless networks. IEEE Wireless Commun., 9(4):8-27, August 2002.

[120] M. Chiang. Balancing transport and physical layers in wireless multihop networks: Jointly optimal congestion control and power control. IEEE J. Select. Areas Commun., 23(1):104-116, January 2005.

[121] U. Kozat, I. Koutsopoulos, and L. Tassiulas. A framework for crosslayer design of energy-efficient communication with QoS provisioning in multi-hop wireless networks. In Proc. IEEE INFOCOM, volume 2, pages 1446-1456, March 2004

[122] S. Cui, R. Madan, A. Goldsmith, and S. Lall. Cross-layer energy minimization in sensor networks. In Proc. Allerton Conference on Communication, Control, and Computing, September/October 2004.

[123] R. Bhatia and M. Kodialam. On power efficient communication over multi-hop wireless networks: Joint routing, scheduling and power control. In Proc. IEEE INFOCOM, volume 2, pages 1457-1466, March 2004.

[124] Y. Eisenberg, C. Luna, T. Pappas, R. Berry, and A. Katsaggelos. Joint source coding and transmission power management for energy efficient wireless video communications. IEEE Trans. Circuits Syst. Video Technol., 12(6):411-424, June 2002.

[125] M. Gastpar and M. Vetterli. Power, spatio-temporal bandwidth, and distortion in large sensor networks. IEEE J. Select. Areas Commun., 23(4):745-754, April 2005.

[126] J. Yuan and W. Yu. Joint optimization of source coding and power 
allocation in sensor networks. In Proc. 23rd Biennial Symposium on Communications, Kingston, Ontario, Canada, May/June 2006.

[127] S. Cui, R. Madan, A. Goldsmith, and S. Lall. Joint routing, MAC, and link layer optimization in sensor networks with energy constraints. In Proc. IEEE International Conference on Communications (ICC), volume 2, pages 725-729, May 2005.

[128] R. Madan, S. Cui, S. Lall, and A. Goldsmith. Cross-layer design for lifetime maximization in interference limited wireless sensor networks. In Proc. IEEE INFOCOM, volume 3, pages 1964-1975, March 2005.

[129] R. Cruz and A. Santhanam. Optimal routing, link scheduling and power control in multihop wireless networks. In Proc. INFOCOM, volume 1, pages 702-711, March/April 2003.

Satyajayant Misra received his integrated M.Sc. (Tech) Information Systems and M.Sc. (Hons) Physics in June 2003 from the Birla Institute of Technology and Sciences (BITS), Pilani, India. He is currently a Ph.D. student in the Department of Computer Science and Engineering at Arizona State University, Tempe. Satyajayant's research interests include identifying security, privacy, and survivability issues in wireless sensors and ad hoc networks and formulating efficient solutions to handle them. Some of his latest works include schemes for anonymity, secure localization, fault tolerant relay node placement in WSNs, and also multimedia transmission in sensor networks.
Martin Reisslein is an Associate Professor in the Department of Electrical Engineering at Arizona State University, Tempe. He received his Ph.D. in systems engineering from the University of Pennsylvania in 1998. From July 1998 through October 2000 he was a scientist with the German National Research Center for Information Technology (GMD FOKUS), Berlin and lecturer at the Technical University Berlin. He maintains an extensive library of video traces for network performance evaluation, including frame size traces of MPEG-4 and H.263 encoded video, at http: //trace.eas . asu.edu.

Guoliang Xue is a Professor of Computer Science and Engineering at Arizona State University, Tempe. He received his $\mathrm{PhD}$ degree in computer science from the University of Minnesota in 1991, and has published extensively in the areas of QoS provisioning, survivability, and resource allocation in wireless ad hoc and sensor networks. He is an Editor of IEEE Network, Computer Networks, and the IEEE Transactions on Wireless Communications, and a TPC co-chair of IEEE Infocom 2010. 